\title{
SNP- and haplotype-based genome-wide association studies for growth, carcass, and meat quality traits in a Duroc multigenerational population
}

Shuji Sato ${ }^{1 * \dagger}$, Yoshinobu Uemoto ${ }^{1 \dagger}$, Takashi Kikuchi ${ }^{1}$, Sachiko Egawa ${ }^{2}$, Kimiko Kohira ${ }^{1}$, Tomomi Saito ${ }^{1}$, Hironori Sakuma', Satoshi Miyashita², Shinji Arata², Takatoshi Kojima' and Keiichi Suzuki ${ }^{3}$

\begin{abstract}
Background: The aim of the present study was to compare the power of single nucleotide polymorphism (SNP)based genome-wide association study (GWAS) and haplotype-based GWAS for quantitative trait loci (QTL) detection, and to detect novel candidate genes affecting economically important traits in a purebred Duroc population comprising seven-generation pedigree. First, we performed a simulation analysis using real genotype data of this population to compare the power (based on the null hypothesis) of the two methods. We then performed GWAS using both methods and real phenotype data comprising 52 traits, which included growth, carcass, and meat quality traits.

Results: In total, 836 animals were genotyped using the Illumina PorcineSNP60 BeadChip and 14 customized SNPs from regions of known candidate genes related to the traits of interest. The power of SNP-based GWAS was greater than that of haplotype-based GWAS in a simulation analysis. In real data analysis, a larger number of significant regions was obtained by SNP-based GWAS than by haplotype-based GWAS. For SNP-based GWAS, 23 genome-wide significant SNP regions were detected for 17 traits, and 120 genome-wide suggestive SNP regions were detected for 27 traits. For haplotype-based GWAS, 6 genome-wide significant SNP regions were detected for four traits, and 11 genome-wide suggestive SNP regions were detected for eight traits. All genome-wide significant SNP regions detected by haplotype-based GWAS were located in regions also detected by SNP-based GWAS. Four regions detected by SNP-based GWAS were significantly associated with multiple traits: on Sus scrofa chromosome (SSC) 1 at $304 \mathrm{Mb}$; and on SSC7 at 35-39 Mb, 41-42 Mb, and $103 \mathrm{Mb}$. The vertnin gene (VRTN) in particular, was located on SSC7 at $103 \mathrm{Mb}$ and was significantly associated with vertebrae number and carcass lengths. Mapped QTL regions contain some candidate genes involved in skeletal formation (FUBP3; far upstream element binding protein 3) and fat deposition (METTL3; methyltransferase like 3).
\end{abstract}

Conclusion: Our results show that a multigenerational pig population is useful for detecting QTL, which are typically segregated in a purebred population. In addition, a novel significant region could be detected by SNPbased GWAS as opposed to haplotype-based GWAS.

Keywords: Duroc pigs, Haplotype-based GWAS, Known candidate genes, Production traits, SNP-based GWAS

\footnotetext{
* Correspondence: s0sato@nlbc.go.jp

${ }^{\dagger}$ Equal contributors

${ }^{1}$ National Livestock Breeding Center, Nishigo, Fukushima 961-8511, Japan

Full list of author information is available at the end of the article
} 


\section{Background}

Even before performance testing in pig breeding, single nucleotide polymorphisms (SNPs) associated with economically important traits (also referred to as quantitative trait loci $[\mathrm{QTL}])$ can be useful in the selection of young animals. Several local animal experiment stations in Japan have performed closed-line breeding for more than five generations of purebred pigs $[1,2]$. To improve these purebred populations using QTL information, segregation of QTL within a purebred population is necessary. One of the methods to detect segregation of QTL that affect economically important traits is genome-wide linkage-based QTL mapping in closed-line breeding populations as described by Uemoto et al. [3] and Soma et al. [4]. Using a purebred Duroc population comprised of multigenerational pedigree, these researchers detected several QTL and further fine mapping studies revealed significant SNPs in positional candidate genes, such as the stearoyl-CoA desaturase $(S C D)$ gene for fatty acid composition [5] and the leptin receptor (LEPR) gene for fat accumulation [6]. These QTL can be used in the breeding management of purebred populations. Therefore, closed-line breeding population is useful to detect QTL.

Recent studies have reported genetic loci that are associated with complex traits using genome-wide association studies (GWAS) with high-density SNP arrays [7], and have demonstrated the power of GWAS to detect significant QTL in pig populations. This linkage disequilibrium (LD)-based QTL mapping with SNP array gives hope to the prospect of finding more QTL for economically important traits in pig populations. Linkage-based GWAS and LD-based GWAS can both detect significant SNPs in LD with common QTL variants that exert medium to high effects; however, LD-based GWAS permits finer mapping than linkage-based GWAS does [8]. Some studies have drawn comparisons between the methods employed for linkage-based GWAS and LDbased GWAS using SNP arrays in half-sib or $F_{2}$ intercross pig populations [9-11], and found that the results of LD-based GWAS were generally consistent with those of linkage-based GWAS. However, to our knowledge, a comparison of both methods has not been performed in a purebred population comprising multigenerational pedigree. In addition, novel candidate regions might be detectable in such populations.

The purpose of the present study was (1) to compare the power between SNP-based GWAS and haplotypebased GWAS for QTL detection in simulation and real phenotype data analyses, and (2) to detect novel candidate genes affecting economically important traits. We used real genotype data of a purebred Duroc population comprising seven-generation pedigree in simulation analysis. These genotypes and 52 quantitative traits related to growth, carcass, and meat quality were used in real phenotype data analysis. We performed the linear mixed model association test at each SNP locus for LD-based GWAS (referred to as SNP-based GWAS), and at each haplotype locus for linkage-based GWAS (referred to as haplotype-based GWAS). Haplotypes were constructed based on pedigree and LD information [12].

\section{Methods}

\section{Experimental animals}

All procedures involving animals followed the Guidelines for the Care and Use of Laboratory Animals established by the National Livestock Breeding Center. A total of 836 Duroc purebred pigs at the National Livestock Breeding Center in Japan were used for GWAS. This population comprised one family from the first to the seventh generation; therefore, all individuals were related. Table 1 shows the number of animals by generation. The pigs had been selected on the basis of average daily gain (DG) from 30 to $105 \mathrm{~kg}$ of body weight, ultrasonically measured loin eye muscle area (LEA), backfat thickness (BF) at $105 \mathrm{~kg}$ weight, and intramuscular fat (IMF) content. The set breeding goals were as follows: $1100 \mathrm{~g} / \mathrm{d}$ (for DG), $36 \mathrm{~cm}^{2}$ (for LEA), $2.6 \mathrm{~cm}$ (for BF), and 6 \% (for IMF content). DG, LEA, and BF were measured in all pigs, and IMF content was measured in slaughtered sib-tested pigs. Boars (16) and gilts (22) were mated in the first generation, and 22 gilts from among their offspring and nine boars were then mated in the second generation. Pigs in the first and second generations were regarded as the base population. Closed-line breeding was performed from the third to the seventh generation, and phenotypes were determined in each generation. Pig in each generation were mated to account for inbreeding and to prevent rapid loss of genetic diversity, and a new generation was obtained each year. The inbreeding coefficient was estimated by the algorithm of Meuwissen and Luo [13], and the inbreeding coefficient of animals in each generation is shown in Additional file 1: Figure S1. Growth traits were measured in all pigs, and carcass and meat quality traits were measured in slaughtered sib-tested pigs. The selection method used in

Table 1 Population number by generation

\begin{tabular}{|c|c|c|c|c|c|c|}
\hline \multirow[b]{2}{*}{ Generation } & \multicolumn{2}{|c|}{ Male } & \multicolumn{2}{|c|}{ Female } & \multicolumn{2}{|c|}{ Total } \\
\hline & $\overline{\text { All }}$ & Sib-tested & $\overline{A l l}$ & $\overline{\text { Sib-tested }}$ & $\overline{A l l}$ & Sib-tested \\
\hline 1 & 16 & 0 & 22 & 0 & 38 & 0 \\
\hline 2 & 19 & 10 & 40 & 11 & 59 & 21 \\
\hline 3 & 42 & 19 & 47 & 10 & 89 & 29 \\
\hline 4 & 67 & 31 & 86 & 26 & 153 & 57 \\
\hline 5 & 73 & 40 & 86 & 21 & 159 & 61 \\
\hline 6 & 71 & 39 & 86 & 27 & 157 & 66 \\
\hline 7 & 80 & 44 & 101 & 30 & 181 & 74 \\
\hline Total & 368 & 183 & 468 & 125 & 836 & 308 \\
\hline
\end{tabular}


closed-line breeding was based on the methods of Suzuki et al. [1]. Breeding values of DG, LEA, BF, and IMF content were predicted by multi-trait animal model best liner unbiased prediction (BLUP), and aggregate breeding values were calculated by multiplying the relative economic weights by the estimated breeding value of each trait. The respective economic weights for DG, LEA, BF, and IMF content were assumed as $0.000,0.882,-1.778$, and 4.450 , respectively. The aggregate breeding values were used as an indicator for selection in the breeding program.

\section{Genotyping}

Genomic DNA of 836 animals was extracted from ear tissue of 10-day-old pigs using proteinase $\mathrm{K}$ and the phenol method. Sample DNA was quantified and genotyped using the Illumina PorcineSNP60 BeadChip (Illumina, San Diego, CA, USA) according to the manufacturer protocol. Image data were analyzed with the iScan (Illumina) and genotype data were then called using the genotyping module contained in the GenomeStudio software (Illumina). Autosomal chromosomes were used and SNP quality control was assessed using the PLINK software [14]. The exclusion criteria for SNPs were minor allele frequency (MAF) $<0.01$, call rate $<0.95$, and Hardy-Weinberg equilibrium test $<0.001$. The exclusion criteria for animals was call rate $<0.95$.

Fourteen SNPs were chosen based on the current knowledge of physiological roles or the known candidate genes affecting pig meat quality and carcass traits, as shown in Table 2. These SNPs were genotyped by polymerase chain reaction-restriction fragment length polymorphism (PCR-RFLP), TaqMan SNP assay, or fragment length analysis. Marker information, such as associated production trait, PCR primers, reporters, and restriction enzymes for discriminating sequence variations are presented in Additional file 2: Table S1. Following quality control measures, the final data set included 831 individuals, which were genotyped at 38,128 SNPs $(38,114$ SNPs in the SNP array and 14 SNPs in the known candidate genes) and which were available for GWAS.

\section{LD information}

We estimated LD coefficient $\left(r^{2}\right)$ values, which are a measure of LD, for all pairs of SNPs less than $10 \mathrm{Mb}$ apart by PLINK software [14]. Average $r^{2}$ values for a given intermarker distance, with marker distances grouped in $1 \mathrm{~kb}$ bins, were estimated in each autosome, and the average $r^{2}$ values among chromosomes were then calculated. Average $r^{2}$ values were then plotted against intermarker distance, as shown in Additional file 3: Figure S2. The results showed that moderate LD (the $r^{2}$ value $=0.20$ ) extended to about $1.0 \mathrm{Mb}$ in this population. The threshold for useful LD was assumed to be $r^{2}$ value $\geqq 0.20$ for the application of GWAS in this study, and the extent of LD (1.0 Mb) was applied for simulation study and QTL detection.

\section{SNP-based GWAS}

We performed an SNP-based association study to detect significant SNPs. The adjusted phenotypes were first obtained by the following mixed model:

$$
\mathbf{y}=\mathbf{X b}+\mathbf{Z u}+\mathbf{e}
$$

where $\mathbf{y}$ is the observation; $\mathbf{X}$ and $\mathbf{Z}$ are the design matrices for fixed and random effects, respectively; $\mathbf{b}$ represents sex (three classes; boar, barrow, and gilt) and generation (seven classes) effects as a fixed effect; this generation effect is the environmental effect at each

Table 2 Genetic markers associated with production trait

\begin{tabular}{|c|c|c|c|}
\hline Chromosome & Gene symbol/SNPa & Gene name & Trait \\
\hline 1 & CYB5A & cytochrome b5 type A (microsomal) & Boar taint \\
\hline 1 & MC4R & melanocortin-4 receptor & Growth \\
\hline 2 & CAST & calpastatin & Meat quality \\
\hline 4 & FABP4 & fatty acid binding protein 4, adipocyte & Fat \\
\hline 6 & ALGA0113531 & uncharacterized LOC102157459 & Boar taint \\
\hline 6 & FTO & fat mass and obesity-associated & Fat \\
\hline 6 & HFABP & heart fatty acid binding protein & Fat \\
\hline 6 & $\angle E P R$ & leptin receptor & Fat \\
\hline 6 & PIK3C3 & phosphoinositide-3-kinase, class 3 & Growth \\
\hline 7 & VRTN & vertnin & Thoracic vertebrae number \\
\hline 8 & CCKAR & cholecystokinin type A receptor & Growth \\
\hline 14 & GPR120 & free fatty acid receptor 4 & Growth \\
\hline 14 & CYP2E1 & cytochrome P450, family 2, subfamily E, polypeptide 1 & Boar taint \\
\hline
\end{tabular}

${ }^{\text {a }}$ olymorphisms detailed information are shown in Additional file 2: Table S1 
generation; $\mathbf{u}$ represents polygenic effects distributed as $N\left(0, \mathbf{A} \sigma_{u}^{2}\right)$; and $\mathbf{e}$ represents the residual effect distributed as $N\left(0, \mathbf{I} \sigma_{e}^{2}\right)$. A is the numerator relationship matrix, $\sigma_{u}^{2}$ is polygenic variance, I is the identity matrix, and $\sigma_{e}^{2}$ is residual variance. The ASREML program [15] was used to estimate all effects, and the adjusted phenotypes $\left(\mathbf{y}_{\text {adj }}\right)$ were then derived by

$$
\mathbf{y}_{\mathbf{a d j}}=\mathbf{y}-\mathbf{X} \hat{\mathbf{b}}
$$

The adjusted phenotypes were then used as the dependent traits in a linear mixed model approach for each SNP:

$$
\mathbf{y}_{\text {adj }}=\beta_{i} \mathbf{w}_{i}+\mathbf{u}+\mathbf{e}_{i}^{\prime}
$$

where $\beta_{i}$ is the allele substitution effect; $\mathbf{w}_{i}$ is a vector of SNP genotypes (coded as 0,1 , or 2 for the homozygote, heterozygote, and the other homozygote, respectively); and $\mathbf{e}_{i}{ }_{i}$ is the random residual at the $i$-th SNP distributed as $N\left(0, \mathbf{I} \sigma_{e}^{2}\right)$. The regression coefficient and $p$-values tested by the Wald test were obtained by Genome-wide mixed-model association (GEMMA) software [16]. The proportion of phenotypic variance explained by the $i$-th SNP effects was calculated using the formula:

$$
\text { Proportion }_{i}=\frac{2 p_{i}\left(1-p_{i}\right) \beta_{i}^{2}}{V_{P}}
$$

where $p_{i}$ is MAF of the $i$-th SNP and $V_{P}$ is the adjusted phenotypic variance [17].

\section{Haplotype-based GWAS}

Haplotype-based GWAS was performed on the basis of pedigree and LD information. Haplotypes were constructed using the hidden Markov model with DualPHASE [12], which assumes the number of ancestral haplotype states (K). A compromise value of $\mathrm{K}=20$ given the simulation considered [12] was used in the present study. The haplotype-based association analysis was then conducted using a linear mixed model using the GLASCOW software [18]. The method by GLASCOW software is composed of two steps: the residuals solved by linear mixed model without haplotype effect is firstly obtained, and the residuals are then used to test significance of association between the haplotype effect and the phenotype at each tested position. Adjusted phenotypes in SNP-based GWAS were used as the dependent traits in the model as follows:

$$
\mathbf{y}_{\text {adj }}=\mathbf{1}_{\mathbf{n}} \mu+\mathbf{H}_{i} \mathbf{h}_{i}+\mathbf{u}+\mathbf{e}_{i}^{\prime}
$$

where $\mathbf{1}_{\mathbf{n}}$ is a vector of $n$ ones; $n$ is the number of animals with phenotype; $\mu$ is the mean; $\mathbf{h}_{\mathbf{i}}$ is a vector of haplotype random effect distributed as $N\left(0, \mathbf{I} \sigma_{h}^{2}\right) ; \mathbf{H}_{\mathrm{i}}$ is the incidence matrix of haplotype genotypes for the individuals at the $i$-th haplotype locus; The element of $\mathbf{H}_{\mathrm{i}}$
$\left(H_{j k}\right)$ is equal to the number of copies (from 0 to 2 ) of ancestral hapotype $k$ carried by $j$-th animal; $\sigma_{h}^{2}$ is the haplotypic variance; and the covariance between ancestral haplotype effects is assumed to be zero. For this analysis, the polygenic effects $(\mathbf{u})$ distributed as $N\left(0, \mathbf{A} \sigma_{u}^{2}\right)$ and the residual effects $\left(\mathbf{e}_{\mathrm{i}}^{\prime}\right)$ distributed as $N\left(0, \mathbf{I} \sigma_{e}^{2}\right)$ were derived in a similar manner as they were for the SNPbased GWAS. Using the GLASCOW software, a test statistics $T$ based on the score tests was calculated at every tested position as follows;

$$
T=0.5\left(\mathbf{y}_{\mathbf{a d j}}-\mathbf{1}_{\mathbf{n}} \mu-\mathbf{u}\right)^{\prime} \mathbf{H}_{i} \mathbf{H}_{i}{ }^{\prime}\left(\mathbf{y}_{\mathbf{a d j}}-\mathbf{1}_{\mathbf{n}} \mu-\mathbf{u}\right)
$$

The $T$ score can be interpreted as a sum over the ancestral haplotypes groups of the sum of the squared difference between observed (i.e., $\mathbf{y}_{\text {adj }}$ ) and expected (i.e., $\mathbf{1}_{\mathbf{n}} \mu+\mathbf{u}$ ) values of the phenotypes for each group. The significance of the $T$ scores can be obtained using the distribution of the $T$ score under the null hypothesis $\left(\sigma_{h}^{2}=0\right)$ that could be approximated using a gamma distribution. Parameters of the gamma distribution were estimated for each tested position.

\section{QTL detection}

For both methods, the Bonferroni correction was applied to determine the $5 \%$ genome-wide significance thresholds $\left(P=1.31 \times 10^{-6}\right)$. The extent of LD in this population was about $1.0 \mathrm{Mb}$, and the genome-wide significance thresholds defined by the Bonferroni correction was too conservative. Therefore, the genome-wide suggestive threshold was also defined as; $P=5.0 \times 10^{-5}$. SNP maps were updated according to the SNPchiMp v.2 database [19] and the Sscrofa 10.2 assembly. The positional candidate genes within the range of $1 \mathrm{Mb}$ bin size of the significant region were scanned using the NCBI2R R-package [20]. $r^{2}$ values between the SNPs of the significant region were calculated, and the haplotype block pattern was visualized using Haploview software [21].

\section{Simulations}

We used real genotype data and pedigree information in simulation analysis. The base phenotypes under the null hypothesis were simulated using a polygenic model with pedigree information [22]. The additive genetic value of $j$-th animal $\left(u_{j}\right)$ was generated based on data from the parent. If both parents were unknown (i.e., the animal is a founder), then $u_{j}$ was derived as follows: $u_{j} \sim N\left(0, \sigma_{u}^{2}\right)$. If both parents were known, $u_{j}$ was derived as follows: $u_{j} \sim N\left(0.5\left(u_{s j}+u_{D j}\right), \quad 0.5\left(1-0.5\left(F_{S j}+F_{D j}\right) \sigma_{u}^{2}\right)\right)$, where $u_{S j}$ and $u_{D j}$ are the additive genetic values of sire and dam of the $j$-th animal, respectively, and $F_{S j}$ and $F_{D j}$ are the inbreeding coefficient for the sire and dam of the $j$-th animal, respectively. The residual value of the $j$-th animal $\left(e_{j}\right)$ was derived from $e_{j} \sim N\left(0, \sigma_{e}^{2}\right)$. The heritability of 
the base phenotype was set at 0.30 , and phenotypic variance was assumed to be 1 . The base phenotype was under the null hypothesis of no phenotype-SNP correlation (i.e., there was no significant effect for GWAS) (Additional file 4: Figure S3), and was used for simulation analysis.

Some missing genotypes were imputed by DualPHASE [12], and were used to generate QTL effects in 100 replicates. In each replicate, we simulated QTL effects under two factors: different QTL MAF categories and QTL heritabilities. Two QTL MAF categories were defined as follows: a low MAF group $(0.01 \leq \mathrm{MAF} \leq 0.10)$ and a high MAF group $(0.10<$ MAF $\leq 0.5)$. Six set values of QTL heritability $(0.01,0.03,0.05,0.07,0.10$, and 0.15$)$ were used. We carried out simulations in various combinations of QTL MAF categories (two analyses) and QTL heritabilities (six analyses). In each replicate, one SNP in the SNP array was randomly selected as the QTL and assigned a QTL effect derived by: $\pm \sqrt{\frac{\sigma_{\text {OTL }}^{2}}{2 p(1-p)}}$; where $\sigma_{\mathrm{QTL}}^{2}$ is the QTL variance and $p$ is the allele frequency of the QTL across all animals. The signs of QTL effects were randomly selected, and the QTL variance was the same as the setting value of QTL heritability, because the phenotypic variance was assumed to be 1 . The QTL effect was then added to the base phenotypic value. An error value was also added to generate a new phenotypic value with a setting value of heritability (0.30). The SNP selected as the QTL was then masked. In each replicate, SNP-based GWAS and haplotype-based GWAS were performed, and the power to achieve $5 \%$ genome-wide significance $\left(P=1.31 \times 10^{-6}\right)$ was calculated as the proportion of replicates with a significant SNP within $\pm 0.5 \mathrm{Mb}$ of the selected QTL. In addition, we also evaluated which method is practical for finer mapping in this population. The power to achieve $5 \%$ genome-wide significance was calculated in three different regions, which ranged from $\pm 0.5 \mathrm{Mb}, \pm 0.5-$ $1.0 \mathrm{Mb}$, and $\pm 1.0-2.0 \mathrm{Mb}$, apart from the selected QTL. In this scenario, the QTL heritability was set to 0.10 .

\section{Phenotypes}

Table 3 summarizes the data of the 52 traits of interest in the present study. Pigs were weighed at birth and at 21 day of age. From this group, pigs (barrows and gilts) that qualified for selection as candidates for the study and for full-sib tests were raised until they attained a body weight of $105 \mathrm{~kg}$. The DG was calculated between 30 and $105 \mathrm{~kg}$ of body weight. Pigs were slaughtered at a live weight of approximately $105 \mathrm{~kg}$. The day before slaughter, body weight, size, and length, including the circumference of the chest (CC) and cannon bone (CCB), the height at the withers (HEIGHT), and the chest depth (CD) and width $(\mathrm{CW})$ of all animals were measured and recorded. Body composition traits comprised ultrasonically measured LEA and BF at half-body length. Structural soundness traits, such as those of the front and rear leg pasterns, were scored at body weights of 30 and $105 \mathrm{~kg}$, based on a scale of 1 (straight) to 5 (soft). Scoring criteria and descriptions are presented in Additional file 5: Figure S4.

Carcasses were scalded and dehaired, and chilled overnight. Carcass measurements were then recorded as follows: weight (CWT); lengths I (CL1), II (CL2), and III (CL3); thickness (CT); and vertebrae number. CL1, CL2, and CL3 denote the lengths from the first cervical vertebra to the pubic bone; from the first rib to the pubic bone; and from the first rib to last lumbar vertebra, respectively. Depth of backfat over the midline was measured with a ruler at the first rib (shoulder: SSFT), at the thinnest depth over the ribs (back: BSFT), and at the first lumbar vertebra (loin: LSFT). One side of the carcass was split between the fourth and fifth ribs (at 45r), and at half-body length (at HBL). The longissimus muscle was traced on acetate paper and the area was determined using computerized morphometric planimetry. Cross sections of each carcass were then recorded photographically. The fat area ratios for subcutaneous fat (SFA), intermuscular fat (IFA), and all fat area (ALLFA) ratios were measured at $45 \mathrm{r}$ and at $\mathrm{HBL}$, using the ImageJ ver 1.48 software [23]. The classification descriptions are presented in Additional file 6: Figure S5.

The weight of each wholesale cut of the remaining side of the carcass was then recorded. Moisture (MOS), IMF, protein (PROT), cooking loss (COOK), water holding capacity (WHC), and shear force value (SF) were measured as described by Okumura et al. [24]. The MOS content was determined by drying approximately $2 \mathrm{~g}$ of a minced sample for $24 \mathrm{~h}$ at $105{ }^{\circ} \mathrm{C}$. Using samples that had already been analyzed for MOS content, IMF content was determined by Soxhlet extraction of the dried samples with diethyl ether for $16 \mathrm{~h}$. The PROT content was determined by the Kjeldahl method, using a nitrogen distillation titration device (2400 Kjeltec Auto Sampler System; FOSS, Hillerod, Denmark). The COOK was determined by placing approximately $50 \mathrm{~g}$ of a cubeshaped sample of meat into a sealed plastic bag, which was then placed in a water bath for $1 \mathrm{~h}$ at $70{ }^{\circ} \mathrm{C}$. For SF determination, at least four pieces that had already been analyzed for COOK were cut (vertical cross section $1 \mathrm{~cm} \times 1 \mathrm{~cm}$ ) parallel to the long axis of the muscle fibers. Each piece was sheared with a Warner-Bratzler shear device attached to an Instron Universal Testing Machine (Model 5542; Instron Japan Co. Ltd., Kanagawa, Japan) with a cross head speed of $200 \mathrm{~mm} / \mathrm{min}$. The WHC was determined by centrifugation of approximately $500 \mathrm{mg}$ of a cube-shaped sample of meat at $2380 \mathrm{~g}$ for $30 \mathrm{~min}$ at $2{ }^{\circ} \mathrm{C}$. The $\mathrm{pH}$ of the longissimus muscle was 
Table 3 Summary statistics of the study subjects

\begin{tabular}{|c|c|c|c|c|c|c|}
\hline Trait & Abbreviation & $\mathrm{N}^{\mathrm{a}}$ & Mean & $S D^{b}$ & Minimum & Maximum \\
\hline \multicolumn{7}{|l|}{ Growth } \\
\hline Average daily gain from 30 to $105 \mathrm{~kg}$ body $\mathrm{wt}, \mathrm{g} / \mathrm{d}$ & DG & 779 & 1094.00 & 112.80 & 750.6 & 1440.0 \\
\hline Ultrasound loin muscle area, $\mathrm{cm}^{2}$ & LEA & 776 & 34.64 & 3.27 & 26.0 & 50.2 \\
\hline Ultrasound backfat thickness, cm & $\mathrm{BF}$ & 776 & 3.17 & 0.56 & 1.8 & 5.0 \\
\hline \multicolumn{7}{|l|}{ Body measurements } \\
\hline Height at withers, $\mathrm{cm}$ & HEIGHT & 779 & 60.97 & 1.98 & 54.4 & 67.4 \\
\hline Length, cm & LENGTH & 779 & 106.00 & 3.38 & 98.0 & 116.0 \\
\hline Front width, cm & FW & 779 & 34.72 & 1.49 & 30.0 & 42.5 \\
\hline Chest width, cm & $C W$ & 779 & 29.88 & 1.51 & 19.0 & 39.2 \\
\hline Rear width, cm & RW & 779 & 32.29 & 1.21 & 28.6 & 38.8 \\
\hline Chest depth, cm & $C D$ & 779 & 35.77 & 1.28 & 24.9 & 39.8 \\
\hline Circumference of chest, $\mathrm{cm}$ & CC & 779 & 111.40 & 3.01 & 99.0 & 120.0 \\
\hline Circumference of cannon bone at front (at $105 \mathrm{~kg}), \mathrm{cm}$ & CCB at F105 & 779 & 18.17 & 0.67 & 16.0 & 20.6 \\
\hline Circumference of cannon bone at rear (at $105 \mathrm{~kg}$ ), cm & CCB at R105 & 779 & 18.91 & 0.62 & 17.0 & 22.4 \\
\hline Circumference of cannon bone at front (at $30 \mathrm{~kg}$ ), cm & CCB at F30 & 681 & 13.70 & 0.64 & 12.0 & 16.2 \\
\hline Circumference of cannon bone at rear (at $30 \mathrm{~kg}$ ), cm & CCB at $\mathrm{R} 30$ & 681 & 14.05 & 0.64 & 12.4 & 16.5 \\
\hline Front leg score at $30 \mathrm{~kg}$ & SCORE at F30 & 718 & 2.70 & 0.54 & 1 & 5 \\
\hline Rear leg score at $30 \mathrm{~kg}$ & SCORE at R30 & 718 & 2.27 & 0.57 & 1 & 5 \\
\hline Front leg score at $105 \mathrm{~kg}$ & SCORE at F105 & 720 & 3.08 & 0.56 & 1 & 5 \\
\hline Rear leg score at $105 \mathrm{~kg}$ & SCORE at R105 & 720 & 2.73 & 0.57 & 1 & 5 \\
\hline \multicolumn{7}{|l|}{ Carcass measurements } \\
\hline Carcass wt, kg & CWT & 302 & 71.76 & 2.53 & 65.1 & 82.0 \\
\hline Carcass yield, \% & CY & 286 & 68.98 & 1.46 & 62.4 & 75.4 \\
\hline Carcass length (1st cervical - pubic), cm & $\mathrm{CL}$ & 302 & 87.64 & 2.19 & 81.5 & 93.0 \\
\hline Carcass length I (1st thoracic - pubic), cm & CL1 & 302 & 72.34 & 1.95 & 67.0 & 77.6 \\
\hline Carcass length II (1st thoracic - last lumbus), cm & $\mathrm{CL2}$ & 302 & 62.76 & 2.09 & 57.5 & 68.3 \\
\hline Carcass length III (Longissimus muscle length), cm & CL3 & 302 & 52.54 & 2.00 & 47.5 & 58.3 \\
\hline Carcass thickness, $\mathrm{cm}$ & $C T$ & 302 & 34.99 & 1.10 & 32.0 & 39.0 \\
\hline \multicolumn{7}{|l|}{ Vertebrae number } \\
\hline Thoracic & TVN & 302 & 14.71 & 0.67 & 13 & 16 \\
\hline Lumbar & LVN & 302 & 6.03 & 0.50 & 5 & 8 \\
\hline Total & - & 302 & 20.74 & 0.59 & 20 & 22 \\
\hline \multicolumn{7}{|l|}{ Subcutaneous fat thickness } \\
\hline Shoulder, cm & SSFT & 302 & 4.00 & 0.58 & 2.4 & 5.8 \\
\hline Back, cm & BSFT & 302 & 2.59 & 0.45 & 1.6 & 4.0 \\
\hline Loin, cm & LSFT & 302 & 3.30 & 0.46 & 2.2 & 6.7 \\
\hline \multicolumn{7}{|l|}{ Longissimus muscle area } \\
\hline at $4-5 \mathrm{rib}, \mathrm{cm}^{2}$ & LEA at $45 r$ & 301 & 17.85 & 3.18 & 9.0 & 31.2 \\
\hline at the middle, $\mathrm{cm}^{2}$ & LEA at HBL & 302 & 36.43 & 4.49 & 26.5 & 50.1 \\
\hline \multicolumn{7}{|l|}{ Meat quality } \\
\hline Moisture content, \% & MOS & 302 & 72.72 & 1.16 & 68.8 & 75.3 \\
\hline Intramuscular fat, \% & IMF & 302 & 5.04 & 1.62 & 1.5 & 9.8 \\
\hline Protein content, $\%$ & PROT & 302 & 21.34 & 0.66 & 19.5 & 23.4 \\
\hline Cooking loss, \% & COOK & 301 & 24.28 & 2.63 & 16.0 & 32.5 \\
\hline
\end{tabular}


Table 3 Summary statistics of the study subjects (Continued)

\begin{tabular}{|c|c|c|c|c|c|c|}
\hline Centrifugal water-holding capacity, \% & WHC & 301 & 78.34 & 3.79 & 68.2 & 87.3 \\
\hline Shear force value, $\mathrm{kg} / \mathrm{cm}^{2}$ & SF & 301 & 2.62 & 0.65 & 1.4 & 4.9 \\
\hline $\mathrm{pH}$ & $\mathrm{pH}$ & 301 & 5.64 & 0.23 & 4.9 & 6.3 \\
\hline Lightness of longissimus muscle $\left(L^{*}\right)$ & $M-L^{*}$ & 300 & 51.63 & 3.44 & 43.6 & 65.3 \\
\hline Redness of longissimus muscle ( $a^{*}$ ) & $M-a^{*}$ & 300 & 3.55 & 1.18 & 0.7 & 6.9 \\
\hline Yellowness of longissimus muscle $\left(b^{*}\right)$ & $M-b^{*}$ & 300 & 6.56 & 1.38 & 1.0 & 11.2 \\
\hline Lightness of subcutaneous fat $\left(L^{*}\right)$ & $\mathrm{F}-\mathrm{L}^{*}$ & 301 & 78.34 & 2.35 & 71.6 & 90.1 \\
\hline Redness of subcutaneous fat $\left(a^{*}\right)$ & $\mathrm{F}-\mathrm{a}^{*}$ & 301 & 3.50 & 0.00 & 1.3 & 0.6 \\
\hline Yellowness of subcutaneous fat (b*) & $F-b^{*}$ & 301 & 7.84 & 1.10 & 5.3 & 10.8 \\
\hline \multicolumn{7}{|l|}{ Fat area of carcass cross section at 4-5 rib } \\
\hline Subcutaneous fat area, $\mathrm{cm}^{2}$ & SFA at $45 r$ & 280 & 81.95 & 15.70 & 47.5 & 131.7 \\
\hline Intermuscular fat area, $\mathrm{cm}^{2}$ & IFA at $45 r$ & 280 & 76.81 & 16.27 & 33.2 & 124.8 \\
\hline All fat area, $\mathrm{cm}^{2}$ & ALLFA at $45 r$ & 280 & 158.80 & 27.00 & 84.8 & 235.0 \\
\hline \multicolumn{7}{|c|}{ Fat area of carcass cross section at the middle } \\
\hline Subcutaneous fat area, $\mathrm{cm}^{2}$ & SFA at $\mathrm{HBL}$ & 280 & 64.78 & 14.41 & 24.1 & 116.8 \\
\hline Intermuscular fat area, $\mathrm{cm}^{2}$ & IFA at $\mathrm{HBL}$ & 280 & 55.79 & 13.49 & 23.4 & 101.9 \\
\hline All fat area, $\mathrm{cm}^{2}$ & ALLFA at HBL & 280 & 120.60 & 25.54 & 57.6 & 218.8 \\
\hline
\end{tabular}

${ }^{\mathrm{a}} \mathrm{N}$ number records

${ }^{\mathrm{b}} \mathrm{SD}$ standard deviation

measured using a pH meter (D-51, HORIBA Ltd., Kyoto, Japan) equipped with a needle-type electrode (6252-10D, HORIBA Ltd.). The appearance of meat and fat (lightness $L^{*}$, redness $a^{*}$, and yellowness $\left.b^{*}\right)$ were assessed immediately after cutting, using a Minolta spectrophotometer CM-2002 (Minolta Camera Co. Ltd., Osaka, Japan). All measurements were performed in duplicate, and means and standard errors were calculated.

\section{Results}

\section{Comparison of the two methods in simulation and real} data analysis

The powers to detect QTL by SNP-based GWAS and haplotype-based GWAS in simulation analysis are presented in Fig. 1. With regard to the impact of QTL MAF on QTL detection, the difference in power between SNP-based GWAS and haplotype-based GWAS was evident. For SNP-based GWAS, the similar trend of the results was observed in the power to detect QTL with high and low MAFs. For haplotype-based GWAS, the power to detect QTL with high MAF was greater, as the QTL heritability increased to more than 0.05 . However, the power to detect QTL with low MAF was quite low at all QTL heritabilities (the maximum value of power was 0.03 ). The power of SNP-based GWAS was greater than that of haplotype-based GWAS under all simulation conditions. For SNP-based GWAS, as the QTL heritability increased, the power to detect QTL also increased and was almost constant at higher QTL heritabilities (more than 0.10). In addition, the power to detect QTL with heritability 0.05 was 0.50 in a high-MAF scenario and 0.45 in a low-MAF scenario. Thus, QTL with smaller heritabilities and both MAFs could be detected by SNP-based GWAS. For haplotype-based GWAS, the power to detect QTL with heritability less than 0.05 was very low (less than 0.03) in a high MAF scenario, but increased as the QTL heritability increased to more than 0.05 .

For the practicability of finer mapping by SNP- and haplotype-based GWAS, the power among three different region apart from the selected QTL were calculated by SNP- and haplotype-based GWAS, and are shown in Fig. 2. The power of haplotype-based GWAS in a lowMAF scenario are not shown, because of very low power (see Fig. 1). For SNP-based GWAS, the similar trend of the results was observed in the power to detect QTL with high and low MAFs. The power decreased from the region $(\mathrm{QTL} \pm 0.5 \mathrm{Mb})$ to the region $(\mathrm{QTL} \pm 0.5-1.0 \mathrm{Mb})$ was a greater extent than it did from the region $(\mathrm{QTL} \pm 0.5-$ $1.0 \mathrm{Mb}$ ) to the region $(\mathrm{QTL} \pm 1.0-2.0 \mathrm{Mb})$, and the decreased power was 0.26 and 0.04 , respectively On the other hand, the power of the haplotype-based GWAS showed a constant decrease, and the decreased power of the region of interest was 0.07 and 0.05 , respectively.

In real data analysis, SNP-based GWAS and haplotypebased GWAS were performed for 52 traits related to growth, carcass, and meat quality. We summarized the genome-wide significant and suggestive SNP regions for these traits in Fig. 3 and Additional file 7: Table S2. For SNP-based GWAS, 23 genome-wide significant SNP regions were detected in 17 traits, and 120 genome-wide 


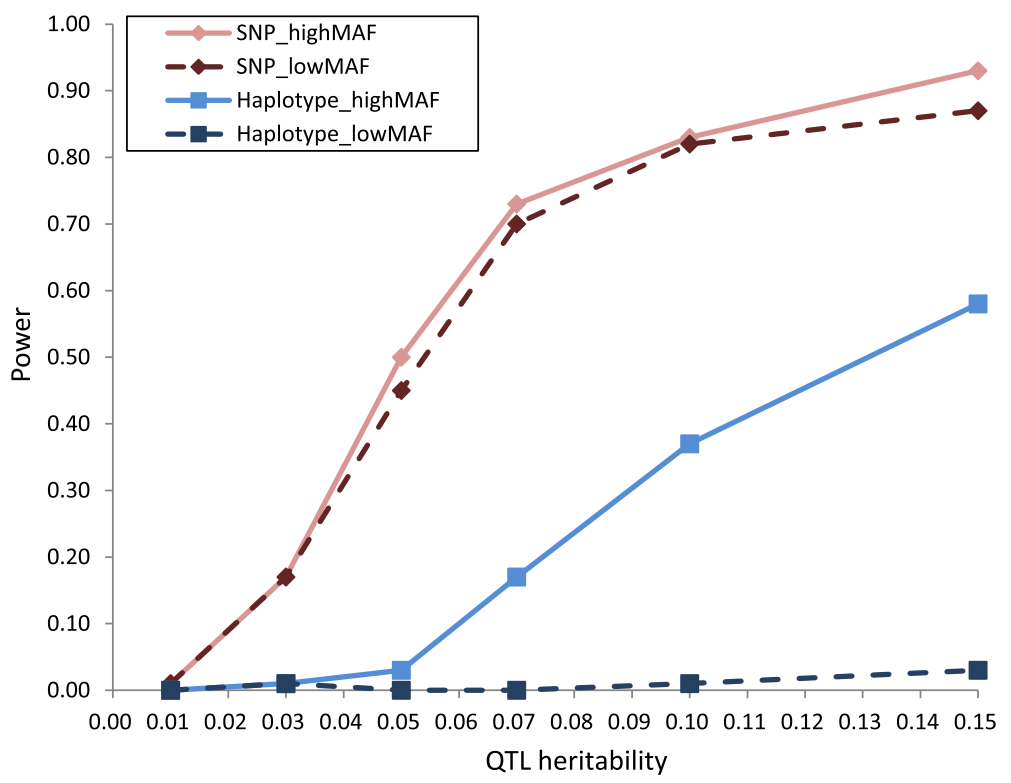

Fig. 1 Power to achieve $5 \%$ genome-wide significance in simulation analysis. The x-axis indicates QTL heritability and the $y$-axis represents the power to detect QTL. Results of varying minor allele frequency (MAF) categories (low and high) and models (SNP-based and haplotype-based genome-wide association studies) are shown

suggestive SNP regions were detected in 27 traits. For haplotype-based GWAS, 6 genome-wide significant SNP regions were detected in four traits, and 11 genome-wide suggestive SNP regions were detected in eight traits. All genome-wide significant SNP regions detected by haplotype-based GWAS were located in regions that were also detected by SNP-based GWAS. Most of the genomewide suggestive SNP regions detected by haplotype-based GWAS were located in regions that were also detected by
SNP-based GWAS. However, four of these regions were detected by haplotype-based GWAS only.

\section{QTL detection}

The SNPs showing genome-wide significant association with growth, body measurements, carcass measurements, and fat area in SNP-based GWAS and haplotypebased GWAS are presented in Table 4. In addition, three regional plots associated with multiple traits are shown

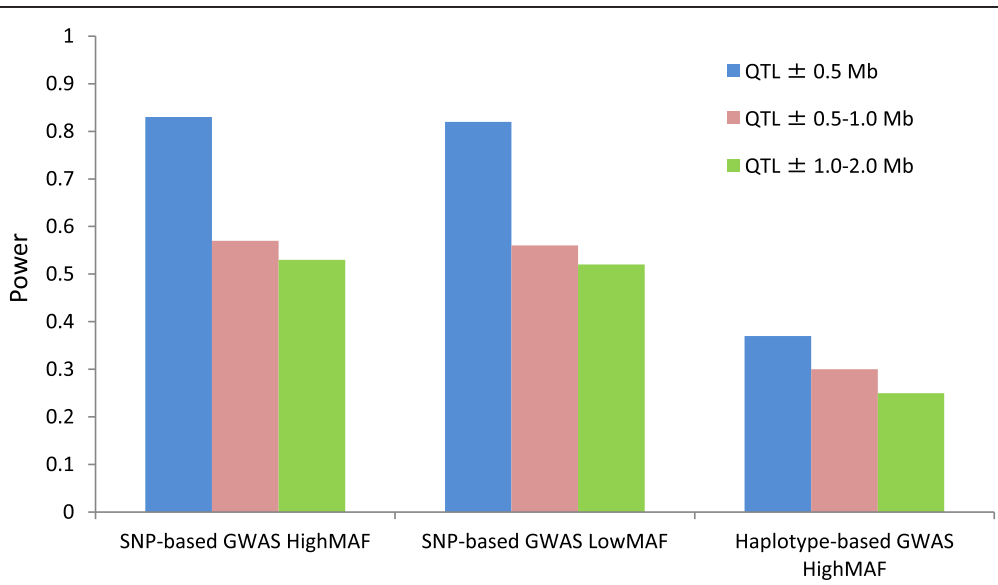

Fig. 2 Power to achieve $5 \%$ genome-wide significance within different ranges around selected QTL in simulation analysis. The $y$-axis represents the power to detect QTL. The results of the SNP-based genome-wide association study (GWAS) in high and low minor allele frequency (MAF) scenarios and those of haplotype-based GWAS in a high MAF scenario are shown. Three different ranges around the selected QTL were evaluated. $\mathrm{QTL} \pm 0.5 \mathrm{Mb}$ : The region ranged from $\pm 0.5 \mathrm{Mb}$ apart from the selected QTL. QTL $\pm 0.5-1.0 \mathrm{Mb}$ : The region ranged from \pm 0.5 to $1.0 \mathrm{Mb}$ apart from the selected QTL. QTL $\pm 1.0-2.0 \mathrm{Mb}$ : The region ranged from \pm 1.0 to $2.0 \mathrm{Mb}$ apart from the selected QTL 
A

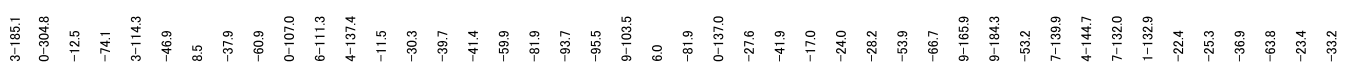

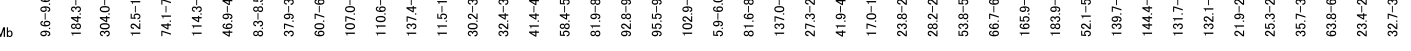

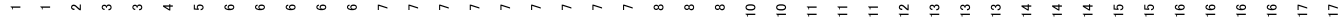

LEA

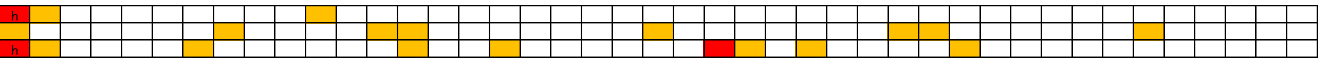

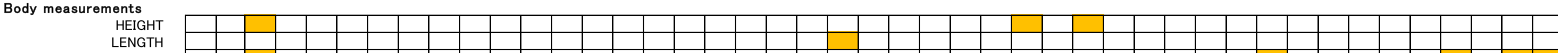

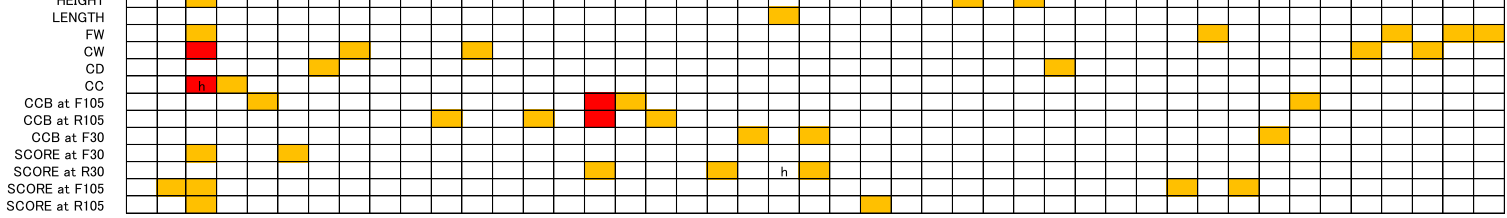

B

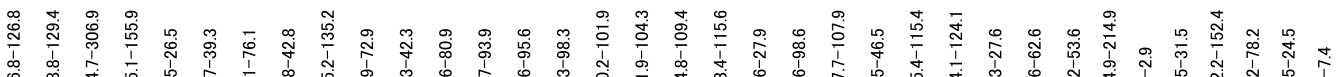

мb

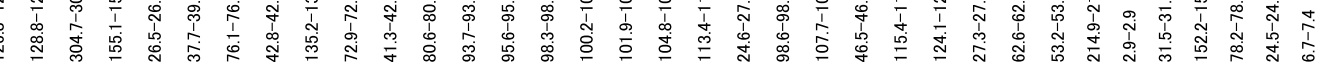

GSCass measurements

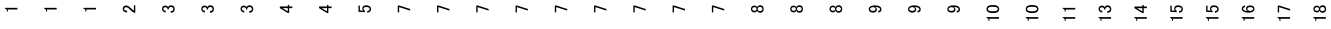

Carcass measurements

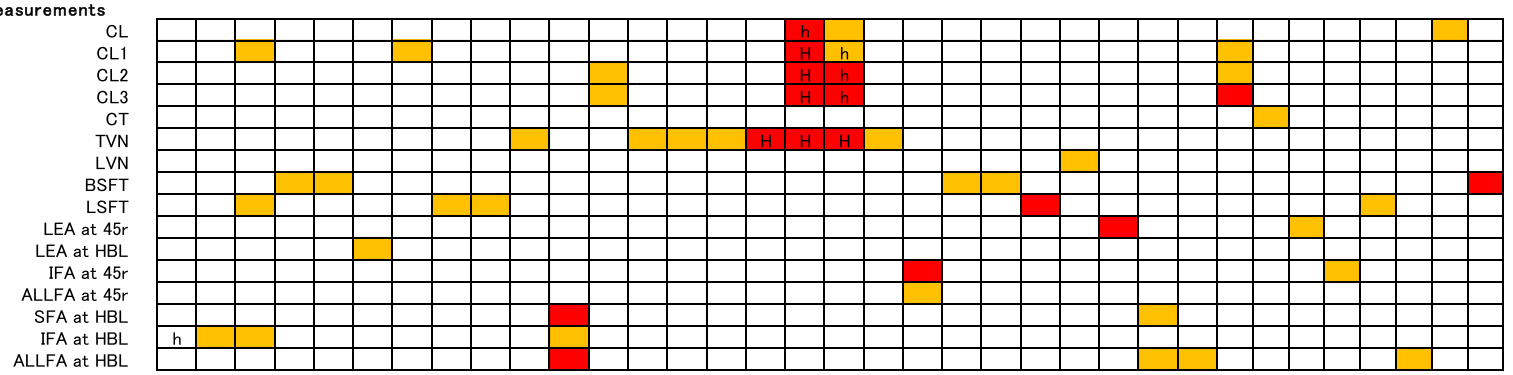

C

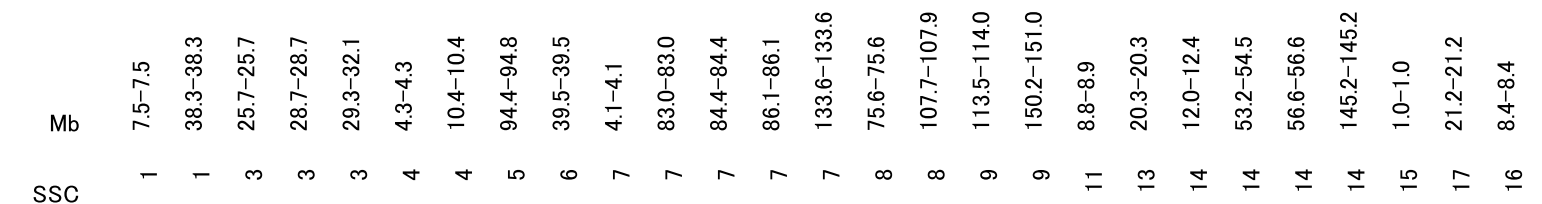

Meat quality

MOS
IMF

IMF
PROT

COOK

WHC

$\mathrm{pH}$

$M-a *$

$M-b *$

F-L*

$\mathrm{F}-\mathrm{a} *$

$\mathrm{F}-\mathrm{b} *$

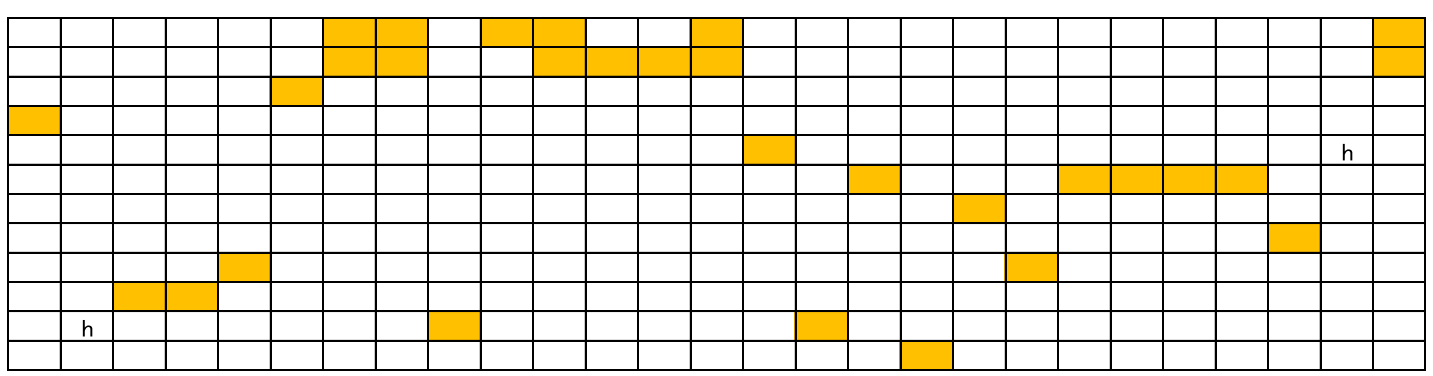

\footnotetext{
No significant associations

Suggestive association $\left(1.31 \mathrm{e}^{-6}<=\mathrm{P}<5 \mathrm{e}^{-5}\right)$ by SNP-based association study

Significance association $(P<1.31 \mathrm{e}-6)$ by SNP-based association study

$h$ Suggestive association $\left(1.31 e^{-} 6<=P<5 e-5\right)$ by haplotype-based association study

$\mathrm{H}$ Significance association $\left(P<1.31 \mathrm{e}^{-6}\right)$ by haplotype-based association study
}

Fig. 3 (See legend on next page.) 
(See figure on previous page.)

Fig. 3 Trait associations across genomic regions analyzed by SNP-based and haplotype-based genome-wide association studies (GWAS). Each row represents a trait, and each column, a genomic region containing SNPS that are genome-wide suggestively or significantly associated with a trait. Only traits with at least one associated SNP and SNPs associated with at least one trait are shown. Each summary shows the results of growth traits (a) carcass traits (b) and meat quality traits (c). SSC, Sus scrofa chromosome; DG, Average daily gain; LEA, Ultrasound loin muscle area; BF, Ultrasound backfat thickness; HEIGHT, Height at withers; FW, Front width; CW, Chest width; CD, Chest depth; CC, Circumference of chest; CCB at F(/R) 30//105), Circumference of cannon bone at front (/Rear) (at $30 \mathrm{~kg} / 105 \mathrm{~kg}$ ); SCORE at F(/R) 30//105), Front (/Rear) leg score at $30 \mathrm{~kg}(/ 105 \mathrm{~kg}) ; \mathrm{CL}$, Carcass length; CL1, Carcass length I; CL2, Carcass length II; CL3, Carcass length III; CT, Carcass thickness; TVN, Thoracic vertebrae number; LVN, Lumbar vertebrae number; BSFT, Subcutaneous fat thickness (Back); LSFT, Subcutaneous fat thickness (Loin); 45r, carcass cross section at fourth-fifth rib; HBL, carcass cross section at half-body length; LEA at 45r, Longissimus muscle area at 45r; LEA at HBL, Longissimus muscle area at HBL; IFA at 45r, Intermuscular fat area at 45r; ALLFA at 45r, All fat area of 45r; SFA at HBL, Subcutaneous fat area at HBL; IFA at HBL, Intermuscular fat area at HBL; ALLFA at HBL, All fat area at HBL; MOS, Moisture; IMF, Intramuscular fat; PROT, Protein; COOK, Cooking loss; WHC, Centrifugal water-holding capacity; SF, Shear force value; M-a*, Redness of longissimus muscle; $M-b^{*}$, Yellowness of longissimus muscle; F-L*, Lightness of subcutaneous fat; F-a*, Redness of subcutaneous fat; F-b*, Yellowness of subcutaneous fat

in Fig. 4. Significant association between rs81352956 on Sus scrofa chromosome (SSC) 1 at $304 \mathrm{Mb}$ and DG $(P=$ $1.22 \times 10^{-8}$ ) was evident in both SNP-based and with haplotype-based analyses (Table 4, Fig. 4a). Each copy of effect allele A at rs81352956 was associated with a decrease in DG of $34.75 \mathrm{~g} / \mathrm{d}$. Moreover, significant associations between rs 81352969 on SSC1 at $304 \mathrm{Mb}$ and BF $\left(P=6.30 \times 10^{-10}\right)$ and CC $\left(P=6.82 \times 10^{-9}\right)$, respectively, were observed in both SNP-based and haplotype-based analyses (Table 4). However, a significant association between rs81352969 on SSC1 at $304 \mathrm{Mb}$ and $\mathrm{CW}(P=$ $1.17 \times 10^{-7}$ ) was only detected with SNP-based GWAS. Each copy of effect allele A at rs81352969 was associated with increases in BF of $0.18 \mathrm{~cm}$; CW of $0.45 \mathrm{~cm}$; and CC of $0.87 \mathrm{~cm}$. Both SNPs, rs81352969 and rs81352956 on SSC1 at $304 \mathrm{Mb}$, were located within close proximity (a distance of $27 \mathrm{~kb}$ ) of each other.

Significant associations of one SNP derived from the vertnin $($ VRTN) gene on SSC7 at $103 \mathrm{Mb}$ with $\mathrm{CL}(P=$ $\left.3.64 \times 10^{-9}\right)$, CL1 $\left(P=1.56 \times 10^{-11}\right)$, CL2 $\left(P=2.96 \times 10^{-14}\right)$, CL3 $\left(P=3.10 \times 10^{-18}\right)$, and thoracic vertebrae number (TVN, $P=9.42 \times 10^{-37}$ ) were observed in both SNP-based and haplotype-based analyses (Table 4, Fig. 4b). Each copy of the $\mathrm{C}$ allele was associated with an estimated increase in the TVN of 0.67 and the proportion of variance in TVN explained by an SNP was about $50 \%$.

Two regions on SSC7, at 35-39 $\mathrm{Mb}$ and 41-42 Mb, respectively, were associated with multiple traits. The region at 35-39 $\mathrm{Mb}$ was associated with $\mathrm{CCB}$ both at the front and rear at $105 \mathrm{~kg}$ body weight $\left(P=3.14 \times 10^{-10}\right.$ and $P=4.38 \times 10^{-8}$, respectively; Table 4, Fig. 4c). Each copy of the $\mathrm{G}$ allele was associated with an estimated increase in CCB of 0.29 and $0.21 \mathrm{~cm}$, respectively. This region was related to the formation of a long-range LD block from 35.0 to $37.5 \mathrm{Mb}$ (see Additional file 8: Figure S6). Significant associations between rs80928067 on SSC7 at 41-42 Mb with SFA at HBL and ALLFA at HBL $\left(P=5.35 \times 10^{-7}\right.$ and $P=5.09 \times 10^{-8}$, respectively) were detected with SNP-based GWAS only (Table 4). Each copy of effect allele G (with MAF 0.030) at rs80928067 was associated with a decrease in SFA at HBL of $16.52 \mathrm{~cm}^{2}$ and in ALLFA at HBL of $30.43 \mathrm{~cm}^{2}$.

Significant SNPs were detected within six regions with SNP-based GWAS only. Significant association between rs81398418 on SSC8 at $25 \mathrm{Mb}$ and IFA at $45 \mathrm{r}(P=$ $4.18 \times 10^{-7}$ ) was detected (Table 4 ), which accounts for $9 \%$ of the phenotypic variance. Each copy of the A allele was associated with an estimated increase in IFA at $45 \mathrm{r}$ of $7.15 \mathrm{~cm}^{2}$. Significant association between rs81257576 on SSC9 at $46 \mathrm{Mb}$ and LSFT $\left(P=7.36 \times 10^{-7}\right)$ was detected. Each copy of the A allele (with MAF 0.017) was associated with an estimated increase in LSFT of $0.65 \mathrm{~cm}$. Significant association between rs81415869 on SSC9 at $124 \mathrm{Mb}$ and LEA at $45 \mathrm{r}\left(P=1.94 \times 10^{-7}\right)$ was detected and accounts for $10 \%$ of the phenotypic variance. Each copy of the G allele (with MAF 0.023) was associated with an estimated increase in LEA at $45 \mathrm{r}$ of $3.98 \mathrm{~cm}^{2}$. Significant association between rs81422289 on SSC10 at $27 \mathrm{Mb}$ and BF $\left(P=7.04 \times 10^{-7}\right)$ was detected. Each copy of the $\mathrm{G}$ allele was associated with an estimated increase in BF of $0.17 \mathrm{~cm}$. Significant association between rs330963199 on SSC11 at $53 \mathrm{Mb}$ and CL3 as longissimus muscle length $\left(P=3.32 \times 10^{-7}\right)$ was detected and accounts for $10 \%$ of the phenotypic variance. Each copy of the $\mathrm{G}$ allele was associated with an estimated decrease in longissimus muscle length of $1.00 \mathrm{~cm}$. Significant association between rs81345146 on SSC18 at $7 \mathrm{Mb}$ and $\operatorname{BSFT}\left(P=4.37 \times 10^{-7}\right)$ was detected and accounts for $10 \%$ of the phenotypic variance. Each copy of the A allele was associated with an estimated decrease in BSFT of $0.19 \mathrm{~cm}$.

No associations were detected in meat quality traits at the genome-wide significance threshold; however, 27 regions were suggested to be associated with meat quality traits (Fig. 3 and Additional file 7: Table S2). We discuss further the regions associated with IMF in greater detail, because this population was generated for the purpose of improving IMF content. Average IMF content was 
Table 4 Top genome-wide significant SNPs associated with growth, body measurements, carcass measurements and fat area

\begin{tabular}{|c|c|c|c|c|c|c|c|c|c|c|}
\hline \multirow[b]{2}{*}{ Trait $^{\mathrm{a}}$} & \multicolumn{8}{|c|}{ Most significant SNP } & \multirow{2}{*}{$\begin{array}{l}\text { Haplotype-based } \\
\text { P-value }^{\mathrm{f}}\end{array}$} & \multirow[t]{2}{*}{ Nearby genes ${ }^{g}$} \\
\hline & $\mathrm{SSC}^{\mathrm{b}}$ & Position $^{\mathrm{c}}$ (bp) & refSNP variation ID & $E A^{d}$ & $\mathrm{EAF}^{\mathrm{d}}$ & $\beta(S E)$ & Proportion $^{e}$ & $P$-value & & \\
\hline \multicolumn{11}{|l|}{ Growth } \\
\hline DG & 1 & $304,667,314$ & rs81352956 & A & 0.454 & $-34.75(6.035)$ & 0.06 & $1.22 \times 10^{-8}$ & $6.71 \times 10^{-6} \mathrm{~h}$ & PRDM12_EXOSC2 \\
\hline \multirow[t]{2}{*}{$\mathrm{BF}$} & 1 & $304,694,455$ & rs81352969 & A & 0.495 & $0.18(0.029)$ & 0.07 & $6.30 \times 10^{-10}$ & $2.26 \times 10^{-5} \mathrm{~h}$ & $A B L 1$ \\
\hline & 10 & $27,636,391$ & rs81422289 & G & 0.227 & $0.17(0.034)$ & 0.04 & $7.04 \times 10^{-7}$ & $3.63 \times 10^{-2}$ & KIF14 \\
\hline \multicolumn{11}{|l|}{ Body measurements } \\
\hline$C W$ & 1 & $304,694,455$ & rs81352969 & A & 0.495 & $0.45(0.084)$ & 0.05 & $1.17 \times 10^{-7}$ & $1.45 \times 10^{-4}$ & $A B L 1$ \\
\hline CC & 1 & $304,694,455$ & rs81352969 & A & 0.495 & $0.87(0.148)$ & 0.06 & $6.82 \times 10^{-9}$ & $3.36 \times 10^{-5} \mathrm{~h}$ & $A B L 1$ \\
\hline CCB at F105 & 7 & $39,512,713$ & rs80892802 & G & 0.171 & $0.29(0.045)$ & 0.07 & $3.14 \times 10^{-10}$ & $1.75 \times 10^{-3}$ & DNAH8 \\
\hline CCB at R105 & 7 & $39,089,506$ & rs196955082 & G & 0.249 & $0.21(0.039)$ & 0.05 & $4.38 \times 10^{-8}$ & $1.19 \times 10^{-3}$ & BTBD9 \\
\hline \multicolumn{11}{|l|}{ Carcass measurements } \\
\hline $\mathrm{CL}$ & 7 & $103,457,401$ & VRTN & C & 0.368 & $1.13(0.187)$ & 0.14 & $3.64 \times 10^{-9}$ & $3.36 \times 10^{-6} \mathrm{~h}$ & VRTN \\
\hline CL1 & 7 & $103,457,401$ & VRTN & C & 0.368 & $1.13(0.162)$ & 0.18 & $1.56 \times 10^{-11}$ & $4.87 \times 10^{-8} \mathrm{H}$ & VRTN \\
\hline \multirow[t]{2}{*}{$\mathrm{CL} 2$} & 7 & $103,457,401$ & VRTN & C & 0.368 & $1.37(0.172)$ & 0.22 & $2.96 \times 10^{-14}$ & $2.22 \times 10^{-9} \mathrm{H}$ & VRTN \\
\hline & 7 & $107,279,922$ & rs80977788 & G & 0.267 & $-0.97(0.196)$ & 0.09 & $1.20 \times 10^{-6}$ & $1.25 \times 10^{-5} \mathrm{~h}$ & NRXN3 \\
\hline \multirow[t]{3}{*}{$\mathrm{CL3}$} & 7 & $103,457,401$ & VRTN & C & 0.368 & $1.48(0.159)$ & 0.27 & $3.10 \times 10^{-18}$ & $1.94 \times 10^{-11} \mathrm{H}$ & VRTN \\
\hline & 7 & $106,308,596$ & rs80813652 & G & 0.311 & $1.02(0.177)$ & 0.12 & $2.14 \times 10^{-8}$ & $1.55 \times 10^{-5} \mathrm{~h}$ & LOC102158165 \\
\hline & 11 & $53,204,914$ & rs330963199 & G & 0.253 & $-1.00(0.191)$ & 0.10 & $3.32 \times 10^{-7}$ & $1.31 \times 10^{-2}$ & LOC102167198 \\
\hline \multirow[t]{3}{*}{ TVN } & 7 & $101,863,838$ & rs80966250 & G & 0.349 & $-0.35(0.059)$ & 0.13 & $9.93 \times 10^{-9}$ & $4.44 \times 10^{-16} \mathrm{H}$ & LOC102164420_LOC102164550 \\
\hline & 7 & $103,457,401$ & VRTN & C & 0.368 & $0.67(0.046)$ & 0.50 & $9.42 \times 10^{-37}$ & $4.44 \times 10^{-16} \mathrm{H}$ & VRTN \\
\hline & 7 & $106,308,596$ & rs80813652 & G & 0.311 & $0.44(0.056)$ & 0.20 & $5.17 \times 10^{-14}$ & $2.67 \times 10^{-9} \mathrm{H}$ & LOC102158165 \\
\hline BSFT & 18 & $7,107,781$ & rs81345146 & A & 0.361 & $-0.19(0.037)$ & 0.10 & $4.37 \times 10^{-7}$ & $7.80 \times 10^{-4}$ & $z Y X$ \\
\hline LSFT & 9 & $46,466,374$ & rs81257576 & A & 0.017 & $0.65(0.129)$ & 0.07 & $7.36 \times 10^{-7}$ & $4.41 \times 10^{-1}$ & ZBTB16_NNMT \\
\hline LEA at $45 r$ & 9 & $124,098,143$ & rs81415869 & G & 0.023 & $3.98(0.746)$ & 0.10 & $1.94 \times 10^{-7}$ & $8.35 \times 10^{-1}$ & LOC102162561_LOC100524389 \\
\hline \multicolumn{11}{|c|}{ Fat area of carcass cross section at $4-5$ rib } \\
\hline IFAat $45 r$ & 8 & $25,653,506$ & rs81398418 & A & 0.279 & $7.15(1.380)$ & 0.09 & $4.18 \times 10^{-7}$ & $1.14 \times 10^{-4}$ & LOC100624133_LOC102162593 \\
\hline \multicolumn{11}{|c|}{ Fat area of carcass cross section at the middle } \\
\hline SFA at HBL & 7 & $41,720,015$ & rs80928067 & G & 0.030 & $-16.52(3.219)$ & 0.11 & $5.35 \times 10^{-7}$ & $5.11 \times 10^{-1}$ & LOC100737927 \\
\hline ALLFA at HBL & 7 & $41,720,015$ & rs80928067 & G & 0.030 & $-30.43(5.433)$ & 0.13 & $5.09 \times 10^{-8}$ & $5.41 \times 10^{-1}$ & LOC100737927 \\
\hline
\end{tabular}

${ }^{a}$ Abbreviations of trait are shown in Table

bSSC Sus Scrofa chromosome

CPosition for genome build 10.2
${ }^{\mathrm{d}} E A$ effect allele, EAF effect allele frequency

'The proportion of phenotypic variance explained by the SNP effects

${ }^{f}$ The results of haplotype-based association study are indicated by $\mathrm{h}=$ suggestive, $\mathrm{H}=$ significant difference

${ }^{9}$ Nearby genes are bolded if SNP is within the reference gene 


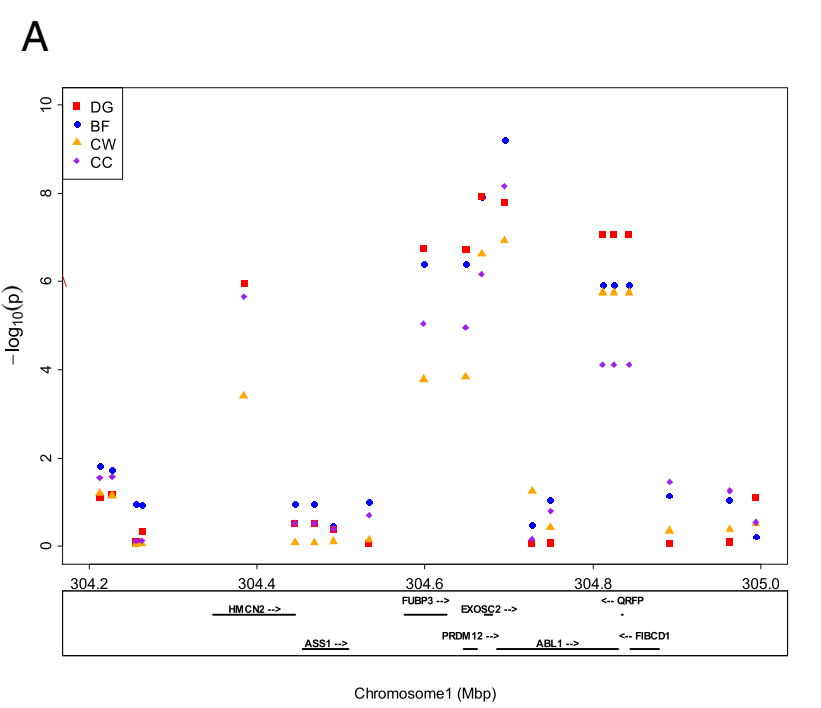

B
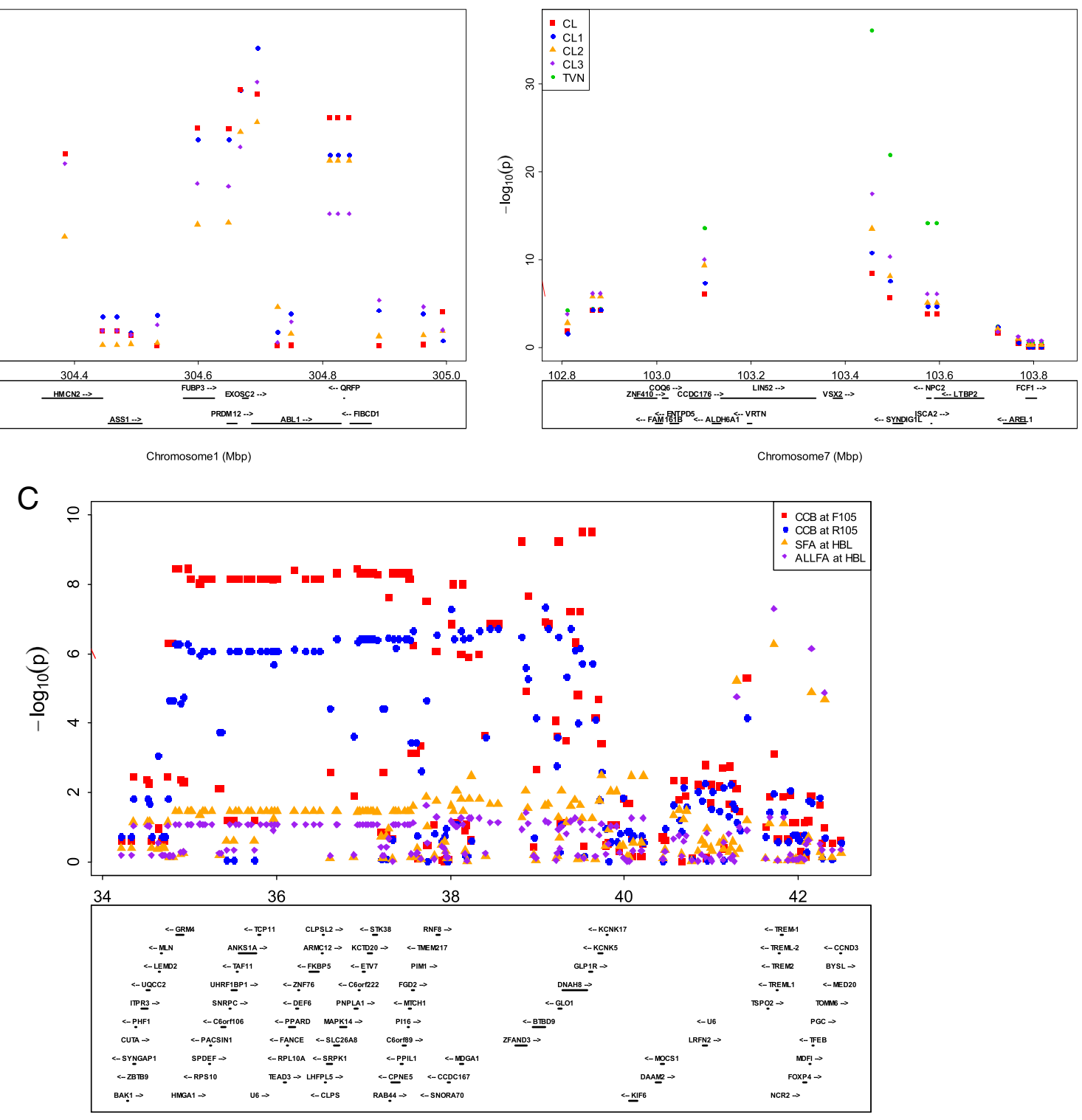

Chromosome7 (Mbp)

Fig. 4 Regional plots of several loci associated with 13 traits. The $x$-axis indicates the Mb, and the $y$-axis indicates - $\log _{10}(p$-value). Gene loci and their strands were annotated based on Sscrofa10.2 assembly from the Ensemble database (http://asia.ensembl.org/Sus_scrofa/Info/Index?db=core). Dashed line indicates the threshold of the Bonferroni 5 \% genome-wide significance level. a Plots in chromosome 1 (304.2-305.0 Mb) for average daily gain (DG), backfat thickness (BF), chest width (CW), and circumference of chest (CC). b Plots in chromosome 7 (102.8-103.9 Mb) for Carcass length (CL), Carcass length I (CL1), Carcass length II (CL2), Carcass length III (CL3), and Thoracic vertebrae number (TVN). c Plots in chromosome 7 (34.2-42.5 Mb) for circumference of cannon bone at front (CCB at F105), circumference of cannon bone at rear (CCB at R105), Subcutaneous fat area of carcass cross section at half-body length (SFA at HBL), and All fat area of carcass cross section at half-body length (ALLFA at HBL)

$5.04 \%(\mathrm{SD}=1.62)$ with a minimum of $1.5 \%$ and a maximum of $9.8 \%$ in this study. The mean breeding value of IMF content in each generation was as follows: generation $1(\mathrm{G} 1)=-0.000 ; \mathrm{G} 2=-0.003 ; \mathrm{G} 3=0.075 ; \mathrm{G} 4=-0.064$; $\mathrm{G} 5=0.230 ; \mathrm{G6}=0.581$; and $\mathrm{G} 7=1.107$. Suggestive association between rs80793147 on SSC4 at $10 \mathrm{Mb}$ and IMF content $\left(P=1.09 \times 10^{-5}\right)$ was detected with SNP-based GWAS only. Each copy of the A allele was associated with an estimated decrease in IMF content of $0.59 \%$. Suggestive association between rs336391107 on SSC7 at $83 \mathrm{Mb}$ and IMF content $\left(P=5.78 \times 10^{-6}\right)$ was detected with SNPbased GWAS only. Each copy of the A allele was 
associated with an estimated decrease in IMF content of $0.58 \%$ (Additional file 7: Table S2). Each of the two SNPs associated with IMF accounted for $7 \%$ of the phenotypic variance.

\section{Discussion \\ Comparison of the two methods in simulation and real data analysis}

In the present study, a comparison of the power to detect QTL between SNP-based GWAS and haplotypebased GWAS was made using simulation and real data. Several significant regions were detected by both methods with real data. For example, the significant region on SSC7 was detected for TVN, and the variant in the VRTN gene was also located in this region. The VRTN gene is the candidate gene that affects TVN [25]; thus, TVN was reliably affected by the detected region. The range of the significant region detected by haplotype-based GWAS was larger than that detected by SNP-based GWAS (Additional file 9: Figure S7A). This trend was similar to that observed in the simulation study. In the simulation study, we also evaluated the power among three regions, which ranged from \pm $0.5 \mathrm{Mb}, \pm 0.5-1.0 \mathrm{Mb}$, and $\pm 1.0-2.0 \mathrm{Mb}$, apart from the selected QTL. As the region of interest grew further apart from the selected QTL, the power of the SNP-based GWAS decreased. In particular, the power decreased from the region of $\pm 0.5 \mathrm{Mb}$ to the region of $\pm 0.5-1.0 \mathrm{Mb}$ to a greater extent than it did from the region of $\pm 0.5-1.0 \mathrm{Mb}$ to the region of $\pm 1.0-2.0 \mathrm{Mb}$. On the other hand, the power of the haplotype-based GWAS showed a constant decrease, as the region of interest grew further apart from the selected QTL. The most significant differences between SNP-based GWAS and haplotype-based GWAS are due to differences in the number of generations considered [8]. Recombination events are scored over a limited number of generations in the pedigree for haplotypebased GWAS, whereas SNP-based GWAS relies on a large number of historical recombination events in past generations. Recombination events with SNPs around the selected QTL can facilitate finer mapping in the QTL region. Previous studies have reported comparisons of both methods in half-sib and $\mathrm{F}_{2}$ intercross pig populations [9-11]. The present study used a multigenerational population, in which the chances of a recombination or segregation event occurring during meiosis would be greater than they would be in a half-sib or $F_{2}$ intercross population. However, finer mapping is still not practical in this population. Therefore, SNP-based GWAS is recommended for fine mapping, provided the significant region can be detected by both methods.

In real data analysis, a larger number of significant regions was detected by SNP-based GWAS than by haplotype-based GWAS. For example, the significant region on SSC7 was detected for ALLFA at HBL by analysis of the SNP-based GWAS. However, the same region was not detected by haplotype-based GWAS (Additional file 9: Figure S7B). This trend was similar to that observed in the simulation study, and the power of SNP-based GWAS was higher than that of haplotype-based GWAS under all simulation conditions. Legarra et al. [10] reported that haplotype-based GWAS yielded lower power and higher rates of false positives in simulation, in comparison to SNP-based GWAS. This finding is consistent with the results of the present study as we also demonstrated the greater power of SNP-based GWAS in comparison to that of haplotype-based GWAS in a multigenerational population. Therefore, a novel region could be detected by SNP-based GWAS.

In the present study, several significant regions were detected in real data by haplotype-based GWAS only. For example, the genome-wide suggestive region on SSC17 was detected for WHC by analysis of the haplotype-based GWAS. However, the significant region was not detected by SNP-based GWAS (Additional file 9: Figure $\mathrm{S} 7 \mathrm{C}$ ). In the simulation study, a significant region detected by haplotype-based GWAS (but not by SNP-based GWAS) was observed in one replicate only under all simulation conditions (Additional file 10: Table S3). This suggested that only SNP-based GWAS or a combination of the two methods could reliably detect QTL, provided one SNP is assumed to be the QTL. In certain cases, linkage-based GWAS can detect rare variants by aggregate analysis as opposed to individual analysis [8]. In the simulations of the present study, only one SNP was assumed to be the QTL, and the phenotypic value was then generated. In that case, the power of the SNP-based GWAS was greater than that of haplotype-based GWAS in a low MAF scenario. Thus, several rare variants in the regions of interest might have caused the observed effects on the trait under investigation. Another possibility is the effect of the copy number variant (CNV), which could not be accounted for in the SNP array. Recent studies have reported the association between $\mathrm{CNV}$ and economically important traits in pigs $[26,27]$. In real data analysis of haplotype-based GWAS of color-sidedness traits in a cattle population, Zhang et al. [18] reported that ancestral haplotypes presented high LD with CNVs that had been previously reported by Durkin et al. [28]; however, the same region was not significant following analysis of SNP-based GWAS. Thus, haplotype-based GWAS can capture variants associated with the $\mathrm{CNV}$, and further study is needed to detect the variant(s) around the significant regions.

\section{QTL detection}

In the population of the present study, four regions were associated with multiple traits. One region on SSC1 at 
$304 \mathrm{Mb}$ that included rs81352956 was associated with $\mathrm{DG}, \mathrm{BF}, \mathrm{CW}$, and CC. This region was in close proximity to the locus at which the far upstream element (FUSE) binding protein 3 (FUBP3) gene (which is involved in c$M y c$ regulation) is located. In humans, this locus is associated with height [29] and bone mineral density [30]. The candidate gene FUBP3 may affect skeletal formation as it relates to both height and bone mineral density. A region on SSC7 at $103 \mathrm{Mb}$ was associated with $\mathrm{CL}$ and TVN. Mikawa et al. [25] reported that the VRTN gene in this region is responsible for the QTL for the TVN in commercial breeds of pigs. Two of nine polymorphic sites, an insertion of the PRE1 element in intron 1 and an SNP in the promoter region, may have effected changes in expression of the VRTN gene at the embryonic stage. Fan et al. [31] reported complete LD between AB554652.1:g.19034A > C in the promoter region and g.20311_20312ins291 in intron 1 as the causal mutation. In the present study, we used the presence or absence of an insertion of the PRE1 element in intron 1 as a marker for simple diagnosis. An allele of VRTN affects the TVN, $\mathrm{CL}$, and the length of the longissimus muscle. These findings are consistent with those of Nakano et al. [32] who reported that VRTN gene polymorphisms greatly contributed to the TVN and CL-related traits. Two regions on SSC7 at 35-39 $\mathrm{Mb}$ and 41-42 $\mathrm{Mb}$ had genome-wide significance with multiple traits. In the region at 35-39 $\mathrm{Mb}$, an LD block from 35.0 to $37.5 \mathrm{Mb}$ was observed, which is consistent with the report of Guo et al. [33]. A long-range LD block in this region and the positions of several genes could interfere with the detection of polymorphism(s) responsible for CCB. The region on SSC7 at $35 \mathrm{Mb}$ was in close proximity to the locus at which the high mobility group AT-hook 1 (HMGA1) gene (which is involved in many cellular processes, including cell growth and differentiation) is located. A QTL for the CCB in Duroc pigs has been reported in this region [34]. In addition, a QTL for limb bone lengths in a Duroc crossed population has been reported by Guo et al. [35] in the same region. In humans, HMGA2 is one of the strong biological candidates for height [36]. Therefore, HMGA1 is possibly also a strong candidate gene that might improve leg strength in Duroc sires that are used in terminal crosses. The region at $41-42 \mathrm{Mb}$ including rs80928067 was associated with SFA at HBL and was in close proximity to the locus at which the triggering receptor expressed on myeloid cells 2 (TREM2) gene (which is involved in osteoclast development and the antiinflammatory response) is located. In humans, this gene is identified as one with transcripts exhibiting differential patterns of expression in abdominal subcutaneous fat between obese and normal pregnant women [37]. In the mouse, TREM2 is required for adipocyte differentiation and promotes adipogenesis by upregulating adipogenic regulators and inhibiting the $\mathrm{Wnt} 10 \mathrm{~b} / \beta$-catenin signaling pathway [38]. Therefore, we suggest that the TREM2 gene may be a candidate for the quantitative trait of fat deposition.

In the present study, six regions had genome-wide significance, and three of those six regions contained potential candidate genes. The region on SSC9 at $124 \mathrm{Mb}$ including rs81415869 was associated with LEA at $45 \mathrm{r}$ and was in close proximity to the locus at which the thiamine pyrophosphokinase (TPK1) gene (which is involved in thiamine metabolism) is located. TPK1 is a cellular enzyme that catalyzes the transfer of the pyrophosphate group from ATP to thiamine, to form thiamine pyrophosphate (TPP). TPP is an active cofactor for enzymes involved in glycolysis and energy generation. In humans, QTL for birth weight have been mapped on chromosome 7, using linkage analysis [39] and TPK1 has been tested as a candidate gene [40]. With respect to birth weight, phenotype was markedly influenced by skeletal muscle mass, and TPK1 may be a candidate gene for the quantitative trait LEA at 45r. The region on SSC10 at $27 \mathrm{Mb}$ including rs81422289 was associated with $\mathrm{BF}$ and was in close proximity to the zinc finger protein 281 (ZNF281) gene, which was predicted to be a potential target of microRNA-33 (miR-33) [41]. In mice, miR-33 coordinates genes that regulate progression of the cell cycle, fatty acid and glucose metabolism, and cholesterol homeostasis [42]. Therefore, we suggest that FUBP3 and ZNF281 may be candidate genes for the quantitative traits DG and BF. The region on SSC18 at $7 \mathrm{Mb}$ including rs81345146 was associated with BSFT and was in close proximity to the locus at which the zyxin $(Z Y X)$ gene (which has $\mathrm{N}$-terminal proline-rich repeats and three copies of LIM (Lin-11, IsI-I, and Mec3) domains in its C-terminal half [43]) is located. Macalma et al. [43] suggested that $Z Y X$ is involved in a number of important signaling pathways that regulate cell differentiation, proliferation, and morphology. Proline-rich and LIM domains interacted with a number of proteins and specific protein partners, respectively. One of those partners, the Homeodomain-interacting protein kinase 2 (HIPK2) gene, identified as an essential regulator of white fat development, might have functions within the adipocyte cell compartment of the skin in the mouse [44]. Therefore, we suggest that $Z Y X$ may be a candidate gene for quantitative trait of fat deposition.

In the present study, we observed no genome-wide significant association with IMF. However, two regions with genome-wide suggestive association contained potential candidate genes. The region on SSC4 at $10 \mathrm{Mb}$ including rs80793147 was associated with IMF content and was in close proximity to the locus at which the ArfGAP with SH3 domain, ankyrin repeat, and $\mathrm{PH}$ domain 1 (ASAP1) gene (also known as differentiation- 
enhancing factor; $D E F-1$ ) is located. DEF-1 is involved in the differentiation of fibroblasts and possibly that of other cell types. In vitro, this gene encoded a Src SH3 binding protein, increased expression of DEF-1, promoted adipogenesis in cultured fibroblast cell lines, and was detected in the adipose tissue of obese and diabetic mice. In addition, the ubiquitous expression pattern of DEF-1 implies that its function is not restricted to adipogenesis [45]. The region on SSC7 at $83 \mathrm{Mb}$ including rs336391107 was associated with IMF content, and was in close proximity to the locus at which the methyltransferase like 3 (METTL3) gene (which is involved in messenger RNA modification of $\mathrm{N}^{6}$-methyladenosine $\left(\mathrm{m}^{6} \mathrm{~A}\right)$ methylation) is located. Adenosine methylation in RNA is a reversible modification that is widespread throughout the transcriptome, and plays an important role in RNA biology, which influences a wide variety of biological pathways and physiological processes [46]. Fustin et al. [47] reported that inhibition of $\mathrm{m}^{6} \mathrm{~A}$ RNAmethylation by METTL3 suppression reduced RNA processing efficiency. In porcine adipocytes, overexpression of METTL3 significantly reduced the intracellular triglyceride content, significantly increased glycerol content in the medium, and significantly downregulated relative mRNA expression of proliferator-activated receptor $\gamma$ and fatty acid synthase genes [48]. METTL3 also enhanced $\mathrm{m}^{6} \mathrm{~A}$ levels and inhibited adipogenesis. Therefore, we suggest that METTL3 and DEF-1 may be candidate genes for the quantitative trait of IMF content.

As we expected that the VRTN is a responsible gene for TVN [25, 31], only one of the 14 known candidate genes, VRTN, was confirmed to have significant association with TVN as well as CL. We were unable to confirm any significant associations, even at a suggestive level, for the other 13 known candidate genes. These findings could be attributed to the fact that the MAFs of candidate genes were low (e.g., the MAF of CYB5A, ALGA0113531, LEPR, and PIK3C3 were 0.072, 0.098, 0.025 , and 0.032 , respectively). The effects of candidate genes depend on the genetic background of the population. Further study is necessary to evaluate the effects of candidate genes in different genetic backgrounds. Nevertheless, we identified multiple novel regions related to growth, carcass, and meat quality traits, which were separate from the regions of known candidate genes. The annotation of these genomic regions exhibited several genes that function in the regulation of $c-M y c$, cell growth and differentiation, thiamine metabolism, and adipogenesis. These findings provide new insights for investigations into altered gene functions that result from modifications of RNA and/or micro-RNA, in addition to DNA polymorphisms. Our results also suggest that the detected region could be useful as a means of improving marker-assisted selection in pigs.

\section{Conclusions}

SNP-based and haplotype-based GWAS were performed in a Duroc multigenerational population. A comparison was drawn between the power of SNP-based GWAS and that of haplotype-based GWAS, using simulation and real data; SNP-based GWAS demonstrated greater power than haplotype-based GWAS in the population under investigation. In real data analysis, larger numbers of significant regions were obtained and some regions had genome-wide significant association with multiple traits. In these significant regions, genes that serve specific functions were located. Among them, FUBP3, a gene implicated in $c-m y c$ regulation, is highlighted as novel candidate gene for skeletal formation associated with average daily gain, and METTL3, a gene implicated in messenger RNA modification, is novel candidate gene for fat deposition associated with IMF content. Further research could identify the causal genes involved in growth, carcass, and meat quality traits.

\section{Ethics (and consent to participate)}

All procedures involving animals followed the Guidelines for the Care and Use of Laboratory Animals established by the National Livestock Breeding Center, and this research project was approved by the laboratory animals committee (23-9) on the National Livestock Breeding Center.

\section{Consent to publish}

Not applicable.

\section{Availability of supporting data and materials}

The datasets supporting the conclusions of this article are included within the article and its additional files.

\section{Additional files}

Additional file 1: Figure S1. Boxplot of inbreeding coefficient in each generation. (PDF $167 \mathrm{~kb}$ )

Additional file 2: Table S1. Genetic markers associated with production traits and related polymorphisms from a study of candidate genes. (XLSX 17 kb)

Additional file 3: Figure S2. Average linkage disequilibrium coefficient $\left(r^{2}\right)$ values plotted against intermarker distance for all autosomal chromosomes. (PDF 407 kb)

Additional file 4: Figure S3. Quantile-quantile plot of the $P$-value for SNP-based genome-wide association study in base phenotype. (PDF 279 kb) Additional file 5: Figure S4. Scoring criteria for feet and leg structure soundness traits. (PDF $320 \mathrm{~kb}$ )

Additional file 6: Figure S5. Classification of fat accumulation of carcass cross sectional image. (PDF $206 \mathrm{~kb}$ )

Additional file 7: Table S2. SNPs showing genome-wide significant and suggestive association with multiple measurements of growth, carcass, and meat quality traits. (XLSX $42 \mathrm{~kb}$ )

Additional file 8: Figure S6. Linkage disequilibrium (LD) of the significant region on chromosome 7. (PDF $415 \mathrm{~kb}$ ) 
Additional file 9: Figure S7. Comparison of the regional plots by SNP-based and haplotype-based genome-wide association studies (GWAS). (PDF $285 \mathrm{~kb}$ )

Additional file 10: Table S3. Number of replicates for detection of significant SNPs in simulation study. (XLSX $10 \mathrm{~kb}$ )

\section{Abbreviations}

ASAP1: ArfGAP with SH3 domain, ankyrin repeat, and PH domain 1; BLUP: best liner unbiased prediction; CNV: copy number variant; DEF-1: differentiationenhancing factor; DG: average daily gain; FUBP3: far upstream element (FUSE) binding protein 3; GEMMA: genome-wide efficient mixed-model association; GWAS: genome-wide association study; HIPK2: homeodomain-interacting protein kinase 2; LD: linkage disequilibrium; LEPR: leptin receptor; LIM: Lin-11, IsI-I, and Mec3; MAF: minor allele frequency; METTL3: methyltransferase like 3; miR33: microRNA-33; $\mathrm{m}^{6} \mathrm{~A}: \mathrm{N}^{6}$-methyladenosine; QTL: quantitative trait locus; SCD: stearoyl-CoA desaturase; SNP: single nucleotide polymorphism; SSC: SUS Scrofa chromosome; TPK1: thiamine pyrophosphokinase; TPP: thiamine pyrophosphate; TREM2: triggering receptor expressed on myeloid cells 2; VRTN: vertnin; ZNF281: zinc finger protein 281; ZYX: zyxin.

\section{Competing interests}

The authors declare that they have no competing interests.

\section{Authors' contributions}

SS designed the experiment, analyzed the data, performed polymorphism analysis, and drafted the manuscript. YU designed the experiment, performed the statistical analysis, simulation analysis, and contributed to writing and improving the manuscript. TKi performed DNA extraction, identified the polymorphisms, and performed the genotyping. SE, SM, and SA designed the resource population and collected samples and phenotypes for the association study. KK, TS, and HS performed analysis of meat quality. TKo and KS designed the experiment and managed the entire project. All authors read and approved the final manuscript.

\section{Acknowledgements}

The authors thank the other investigators and staff who were involved in the long-term generation of the population and who generously provided samples.

\section{Funding}

Funding for PorcineSNP60 BeadChip genotyping was provided by the private association project for stepped-up measures of agricultural competitive positions in 2013 and 2014 from the Ministry of Agriculture, Forestry and Fisheries of Japan.

\section{Author details}

${ }^{1}$ National Livestock Breeding Center, Nishigo, Fukushima 961-8511, Japan. ${ }^{2}$ Miyazaki Branch of National Livestock Breeding Center, Kobayashi, Miyazaki 886-0004, Japan. ${ }^{3}$ Graduate School of Agricultural Science, Tohoku University, Sendai, Miyagi 981-8555, Japan.

Received: 19 January 2016 Accepted: 15 April 2016

Published online: 19 April 2016

\section{References}

1. Suzuki K, Kadowaki H, Shibata T, Uchida H, Nishida A. Selection for daily gain, loin-eye area, backfat thickness and intramuscular fat based on desired gains over seven generations of Duroc pigs. Livest Prod Sci. 2005;97:193-202.

2. Kadowaki H, Suzuki E, Kojima-Shibata C, Suzuki K, Okamura T, Onodera W, et al. Selection for resistance to swine mycoplasmal pneumonia over 5 generations in Landrace pigs. Livest Sci. 2012;147:20-6.

3. Uemoto $Y$, Nagamine $Y$, Kobayashi E, Sato S, Tayama T, Suda $Y$, et al Quantitative trait loci analysis on chromosome 7 for meat production, meat quality, and carcass traits within a Duroc purebred population. J Anim Sci. 2008;86:2833-9.

4. Soma $Y$, Uemoto $Y$, Sato $S$, Shibata $T$, Kadowaki $H$, Kobayashi $E$, et al. Genome-wide mapping and identification of new quantitative trait loci affecting meat production, meat quality, and carcass traits within a Duroc purebred population. J Anim Sci. 2011;89:601-8.

5. Uemoto $Y$, Nakano $H$, Kikuchi $T$, Sato $S$, Ishida M, Shibata $T$, et al. Fine mapping of porcine SSC14 QTL and SCD gene effects on fatty acid composition and melting point of fat in a Duroc purebred population. Anim Genet. 2011:43:225-8.

6. Uemoto Y, Kikuchi T, Nakano H, Sato S, Shibata T, Kadowaki H, et al. Effects of porcine leptin receptor gene polymorphisms on backfat thickness, fat area ratios by image analysis, and serum leptin concentrations in a Duroc purebred population. Anim Sci J. 2012;83:375-85.

7. Sharma A, Leea JS, Dang CG, Sudrajad P, Kim HC, Yeon SH, et al. Stories and Challenges of Genome Wide Association Studies in Livestock - A Review. Asian-Australas J Anim Sci. 2015:28:1371-9.

8. Ott J, Wang J, Leal SM. Genetic linkage analysis in the age of wholegenome sequencing. Nat Rev Genet. 2015;16:275-84.

9. Ai H, Xiao S, Zhang Z, Yang B, Li L, Guo Y, et al. Three novel quantitative trait loci for skin thickness in swine identified by linkage and genome wide association studies. Anim Genet. 2014;45:524-33.

10. Legarra A, Croiseau P, Sanchez MP, Teyssèdre S, Sallé G, Allais S, et al. A comparison of methods for whole-genome QTL mapping using dense markers in four livestock species. Genet Sel Evol. 2015:47:6

11. Qiao R, Gao J, Zhang Z, Li L, Xie X, Fan Y, et al. Genome-wide association analyses reveal significant loci and strong candidate genes for growth and fatness traits in two pig populations. Genet Sel Evol. 2015;47:17.

12. Druet T, Georges M. A hidden Markov model combining linkage and linkage disequilibrium information for haplotype reconstruction and quantitative trait locus fine mapping. Genetics. 2010;184:789-98.

13. Meuwissen THE, Luo Z. Computing inbreeding coefficients in large populations. Genet Sel Evol. 1992;24:305-13.

14. Purcell S, Neale B, Todd-Brown K, Thomas L, Ferreira MA, Bender D, et al PLINK: a tool set for whole-genome association and population-based linkage analyses. Am J Hum Genet. 2007;81:559-75.

15. Gilmour AR, Gogel BJ, Cullis BR, Thompson R. Asreml User Guide Release 3. 0 . Hemel Hempstead: VSN International Ltd; 2009.

16. Zhou X, Stephens M. Genome-wide efficient mixed-model analysis for association studies. Nat Genet. 2012:44:821-4.

17. Falconer DS, Mackay TFC. Introduction to Quantitative Genetics. 4th ed. London: Longman Group; 1996.

18. Zhang Z, Guillaume F, Sartelet A, Charlier C, Georges M, Farnir F, et al Ancestral haplotype-based association mapping with generalized linear mixed models accounting for stratification. Bioinformatics. 2012;28:2467-73.

19. Nicolazzi EL, Nazzicari N, Caprera A, Fojadelli I, Strozzi F, Schnabel RD, et al. SNPchiMp v.2: An Open Access Web Tool for SNP Data Management on Bovine, Porcine and Equine Livestock. Proceedings of the 10th World Congress of Genetics Applied to Livestock Production; 2014. https://asas. org/docs/default-source/wcgalp-proceedingsoral/157_paper_9057_ manuscript 406 0.pdf?sfvrsn=2.

20. Melville S, Melville MS. Package 'NCBI2R'. https://cran.r-project.org/src/ contrib/Archive/NCBI2R/ (2012). Accessed 15 Feb 2013

21. Barrett JC, Fry B, Maller J, Daly MJ. Haploview: analysis and visualization of LD and haplotype maps. Bioinformatics. 2005:21:263-5.

22. Blumer MG. The mathematical theory of quantitative genetics. Oxford: Clarendon; 1980.

23. Rasband WS: Image J, U. S. National Institutes of Health, Bethesda, Maryland USA, http://rsb.info.nih.gov/ij/docs/guide/index.html (1997-2012). Accessed 20 May 2015

24. Okumura T, Saito K, Sowa T, Sakuma H, Ohhashi F, Tameoka N, et al. Changes in beef sensory traits as somatic-cell-cloned Japanese black steers increased in age from 20 to 30. Meat Sci. 2012;90:159-63.

25. Mikawa S, Sato S, Nii M, Morozumi T, Yoshioka G, Imaeda N, et al. Identification of a second gene associated with variation in vertebral number in domestic pigs. BMC Genet. 2011;12:5.

26. Fowler KE, Pong-Wong R, Bauer J, Clemente EJ, Reitter CP, Affara NA, et al. Genome wide analysis reveals single nucleotide polymorphisms associated with fatness and putative novel copy number variants in three pig breeds. BMC Genomics. 2013;14:784

27. Wang L, Xu L, Liu X, Zhang T, Li N, Zhang Y, et al. Copy number variationbased genome wide association study reveals additional variants contributing to meat quality in Swine. Sci Rep. 2015;5:12535.

28. Durkin K, Coppieters W, Drögemüller C, Ahariz N, Cambisano N, Druet T, et al. Serial translocation by means of circular intermediates underlies colour sidedness in cattle. Nature. 2012:482:81-4.

29. Lettre G, Jackson AU, Gieger C, Schumacher FR, Berndt SI, Sanna S, et al. Identification of ten loci associated with height highlights new biological pathways in human growth. Nat Genet. 2008;40:584-91. 
30. Kemp JP, Medina-Gomez C, Estrada K, Pourcain BS, Heppe DHM, Warrington $\mathrm{NM}$, et al. Phenotypic dissection of bone mineral density reveals skeletal site specificity and facilitates the identification of novel loci in the genetic regulation of bone mass attainment. PLoS Genet. 2014;6:e1004423.

31. Fan $Y$, Xing $Y$, Zhang Z, Ai H, Ouyang Z, Ouyang J, et al. A further look at porcine chromosome 7 reveals VRTN variants associated with vertebral number in Chinese and western pigs. PLoS One. 2013;8:e62534.

32. Nakano H, Sato S, Uemoto $Y$, Kikuchi T, Shibata T, Kadowaki H, et al. Effect of VRTN gene polymorphisms on Duroc pig production and carcass traits, and their genetic relationships. Anim Sci J. 2015;86:125-31.

33. Guo YM, Zhang XF, Ai RHS, Ma JW, Huang LS. A joint genomewide association analysis of pig leg weakness and its related traits in an $F_{2}$ population and a Sutai population. J Anim Sci. 2013;91:4060-8.

34. Okumura N, Matsumoto T, Hayashi T, Hirose K, Fukawa K, Itou T, et al. Genomic regions affecting backfat thickness and cannon bone circumference identified by genome-wide association study in a Duroc pig population. Anim Genet. 2012;44:454-7.

35. Guo Y, Hou L, Zhang X, Huang M, Mao H, Chen $\mathrm{H}$, et al. A meta analysis of genome-wide association studies for limb bone lengths in four pig populations. BMC Genet. 2015;16:95.

36. Weedon MN, Lettre RMF, Lindgren CM, Voight BF, Perry JRB, Elliott KS, et al. A common variant of HMGA2 is associated with adult and childhood height in the general population. Nat Genet. 2007;39:1245-50.

37. Bashiri A, Heo HJ, Ben-Avraham D, Mazor M, Budagov T, Einstein FH, et al. Pregnancy complicated by obesity induces global transcript expression alterations in visceral and subcutaneous fat. Mol Genet Genomics. 2014;289:695-705.

38. Park M, Yi J, Kim E, Yoon I, Lee E, Lee H, et al. Triggering receptor expressed on myeloid cells 2 (TREM2) promotes adipogenesis and diet-induced obesity. Diabetes. 2015;64:117-27.

39. Fradin D, Heath S, Lepercq J, Lathrop M, Bougnères P. Identification of distinct quantitative trait loci affecting length or weight variability at birth in humans. J Clin Endocrinol Metab. 2006;91:4164-70.

40. Fradin $D$, Bougneres $P$. Three common intronic variants in the maternal and fetal thiamine pyrophosphokinase gene (TPK1) are associated with birth weight. Ann Hum Genet. 2007;71:578-85.

41. Shao F, Wang $X, Y u$ J, Jiang $H$, Zhu B, Gu Z. Expression of miR-33 from an SREBF2 intron targets the FTO gene in the chicken. PLoS One. 2014;9:e91236.

42. Cirera-Salinas D, Pauta M, Allen RM, Salerno AG, Ramírez CM, ChamorroJorganes A, et al. Mir-33 regulates cell proliferation and cell cycle progression. Cell Cycle. 2012;11:922-33.

43. Macalma T, Otte J, Hensler ME, Bockholt SM, Louis HA, Kalff-Suske M, et al. Molecular characterization of human zyxin. J Biol Chem. 1996;271:31470-8

44. Sjölund J, Pelorosso FG, Quigley DA, DelRosario R, Balmain A. Identification of Hipk2 as an essential regulator of white fat development. Proc Natl Acad Sci U S A. 2014;111:7373-8

45. King F, Hu E, Harris DF, Sarraf P, Spiegelman BM, Roberts TM. DEF-1, a novel Src SH3 binding protein that promotes adipogenesis in fibroblastic cell lines. Mol Cell Biol. 1999:19:2330-7.

46. Meyer KD, Saletore Y, Zumbo P, Elemento O, Mason CE, Jaffrey SR. Comprehensive analysis of mRNA methylation reveals enrichment in 3' UTRs and near stop codons. Cell. 2012;149:1635-46.

47. Fustin J-M, Doi M, Yamaguchi Y, Hida H, Nishimura S, Yoshida M, et al. RNAmethylation-dependent RNA processing controls the speed of the circadian clock. Cell. 2013;155:793-806.

48. Wang $X$, Zhu L, Chen J, Wang Y. mRNA $m^{6} A$ methylation downregulates adipogenesis in porcine adipocytes. Biochem Biophys Res Commun. 2015; 459:201-7.

\section{Submit your next manuscript to BioMed Central and we will help you at every step:}

- We accept pre-submission inquiries

- Our selector tool helps you to find the most relevant journal

- We provide round the clock customer support

- Convenient online submission

- Thorough peer review

- Inclusion in PubMed and all major indexing services

- Maximum visibility for your research

Submit your manuscript at www.biomedcentral.com/submit

) Biomed Central 